\title{
THE MCMULLEN DOMAIN: SATELLITE MANDELBROT SETS AND SIERPINSKI HOLES
}

\author{
ROBERT L. DEVANEY
}

\begin{abstract}
In this paper we describe some features of the parameter planes for the families of rational maps given by $F_{\lambda}(z)=z^{n}+\lambda / z^{n}$ where $n \geq 3, \lambda \in \mathbb{C}$. We assume $n \geq 3$ since, in this case, there is a McMullen domain surrounding the origin in the $\lambda$-plane. This is a region where the corresponding Julia sets are Cantor sets of concentric simple closed curves. We prove here that the McMullen domain in the parameter plane is surrounded by infinitely many simple closed curves $\mathcal{S}^{k}$ for $k=1,2, \ldots$ having the property that:

(1) Each curve $\mathcal{S}^{k}$ surrounds the McMullen domain as well as $\mathcal{S}^{k+1}$, and the $\mathcal{S}^{k}$ accumulate on the boundary of the McMullen domain as $k \rightarrow \infty$.

(2) The curve $\mathcal{S}^{k}$ meets the centers of $\tau_{k}^{n}$ Sierpinski holes, each with escape time $k+2$ where

$$
\tau_{k}^{n}=(n-2) n^{k-1}+1 .
$$

(3) The curve $\mathcal{S}^{k}$ also passes through $\tau_{k}^{n}$ parameter values which are centers of the main cardioids of baby Mandelbrot sets with base period $k$.
\end{abstract}

\section{INTRODUCTION}

Our goal in this paper is to describe some of the interesting features of the parameter plane for the families of rational maps given by

$$
F_{\lambda}(z)=z^{n}+\frac{\lambda}{z^{n}}
$$

where $\lambda$ is a complex parameter and $n \geq 3$ is a positive integer. The reason why $n \neq 1,2$ will be made clear below. We regard these maps as singular perturbations of the simple map $z \mapsto z^{n}$. We call these maps singular perturbations since, when $\lambda=0, F_{0}$ has degree $n$, but when $\lambda \neq 0$, the degree of $F_{\lambda}$ jumps to $2 n$. As we discuss below, the corresponding Julia sets for these maps become much more complex (and interesting) when $\lambda$ is non-zero.

The reason for the interest in such singular perturbations stems from Newton's method: When Newton's method is applied to a family of maps that, at a particular parameter value, has a multiple root, a similar jump in degree and complexity arises as the parameters are varied.

While each of the rational maps $F_{\lambda}$ with $\lambda \neq 0$ has degree $2 n$, in fact, just as in the case of the well-studied quadratic polynomial family, this family forms a natural one-parameter family of maps since there is essentially only one "free" critical orbit for each map. This occurs since each of the $2 n$ non-zero finite critical points of $F_{\lambda}$

Received by the editors July 11, 2006.

2000 Mathematics Subject Classification. Primary 37F45; Secondary 37F20.

Key words and phrases. McMullen domain, Sierpinski curve, Mandelbrot set, Julia set, rational map. 
have orbits that behave symmetrically. Hence the $\lambda$-plane is the natural parameter plane to record the behavior of maps in these families.

As another similarity with the quadratic family, the point at $\infty$ is a superattracting fixed point for each $F_{\lambda}$ when $n>1$, and so it may be the case that the critical orbits all enter the immediate basin of attraction of this fixed point. This may happen in a number of different manners. To describe these different scenarios, we denote the immediate basin of attraction of $\infty$ by $B_{\lambda}$. Since 0 is a pole, there is a neighborhood of 0 that is mapped into $B_{\lambda}$. Now either this neighborhood is itself contained in $B_{\lambda}$, or else 0 lies in a disjoint preimage of $B_{\lambda}$ which we denote by $T_{\lambda}$. In the latter case, we note that $F_{\lambda}$ maps $T_{\lambda}$ in $n$ to 1 fashion onto $B_{\lambda}$ while $F_{\lambda} \mid B_{\lambda}$ is also $n$ to 1 . Hence the only preimages of $B_{\lambda}$ are $B_{\lambda}$ itself and $T_{\lambda}$.

In the quadratic family there is only one way for the critical orbit to escape to $\infty$ : Either the critical point and its entire subsequent orbit lies in the basin of $\infty$ or else no point on this orbit lies in the basin. In contrast, there are three distinctly different manners in which the critical orbit of $F_{\lambda}$ may escape to $\infty$, and this in turn determines three different topological structures for the Julia sets corresponding to these escape parameters. The following theorem describes this trichotomy (see [6]).

Theorem (The Escape Trichotomy).

(1) If one, and hence all, of the critical values of $F_{\lambda}$ lie in $B_{\lambda}$, then the Julia set of $F_{\lambda}$ is a Cantor set.

(2) If one, and hence all, of the critical values lie in $T_{\lambda}$, then the Julia set is a Cantor set of simple closed curves.

(3) If the critical values all lie in preimages of $T_{\lambda}$ under $F_{\lambda}^{j}$ for some $j>0$, then the Julia set is a Sierpinski curve.

A Sierpinski curve is a planar set that is characterized by the following five properties: it is a compact, connected, locally connected and nowhere dense set with two or more complementary domains that are all bounded by simple closed curves that are pairwise disjoint. It is known from work of Whyburn [16] that any two Sierpinski curves are homeomorphic. In fact, they are homeomorphic to the well-known Sierpinski carpet fractal. From the point of view of topology, a Sierpinski curve is a universal set in the sense that it contains a homeomorphic copy of any planar, compact, connected, one-dimensional set. The first example of a Sierpinski curve Julia set was given by Milnor and Tan Lei [13].

Case 2 of the Escape Trichotomy was first observed by McMullen [10], who showed that this phenomenon occurs in each of these families provided that $n \neq 1,2$ and $\lambda$ is sufficiently small.

In Figure 1 we display the parameter plane for the family $F_{\lambda}(z)=z^{3}+\lambda / z^{3}$. The black points in this picture correspond to parameter values for which the critical orbit does not escape to $\infty$. Again in analogy with the quadratic polynomial family, for these parameters, the Julia set is known to be a connected set [1]. The white regions in this picture represent $\lambda$-values for which the critical orbit tends to $\infty$. The exterior region corresponds to parameter values for which the Julia set is a Cantor set; we call this set the Cantor set locus. The small white region in the center of the picture corresponds to parameter values for which the Julia set is a Cantor set of simple closed curves. We call this region the McMullen domain. It is known [3] that there is a unique such domain for each $n \geq 3$ and that this region is a simply connected open set that is bounded by a simple closed curve. All other white regions in this picture correspond to parameters for which the Julia set is 


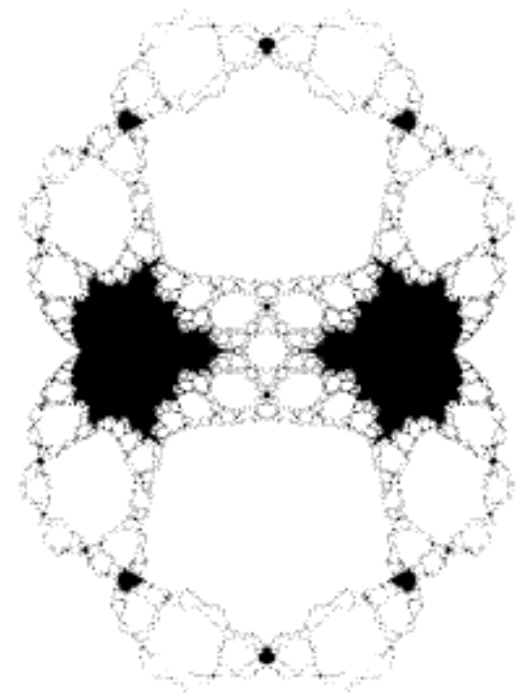

Figure 1. The parameter plane for the family $z^{3}+\lambda / z^{3}$.

a Sierpinski curve. These are called Sierpinski holes. Hence the Julia set of $F_{\lambda}$ is a connected set for all $\lambda$-values except those in the Cantor set locus and the McMullen domain. So we call this set of parameters the connectedness locus.

It is known that there are infinitely many disjoint Sierpinski holes for each of these families [1]. As we show below, there is a unique parameter in each Sierpinski hole for which the orbit of the critical point lands on 0 at some iteration, say iteration $k-1>0$, and therefore on $\infty$ at iteration $k$. We call this $\lambda$-value the center of the Sierpinski hole and $k$ the escape time of the hole. All other parameters in this Sierpinski hole have the property that the critical point has orbit that lands in $B_{\lambda}$ at the escape time iterate. By Whyburn's result, the Julia sets corresponding to any two parameters drawn from a Sierpinski hole are homeomorphic. However, as shown in [6], there exist Sierpinski holes corresponding to each escape time $k \geq 3$, and these have the property that if $\lambda_{1}$ and $\lambda_{2}$ lie in Sierpinski holes with different escape times, then $F_{\lambda_{1}}$ and $F_{\lambda_{2}}$ are not topologically conjugate on their Julia sets. Consequently, even though the topology of these sets is the same, the dynamical behavior on these sets is quite different.

In Figure 1 we see that, in the case $n=3$, there appear to be two large copies of a Mandelbrot set that straddle the positive and negative real axes. These are called the principal Mandelbrot sets for $F_{\lambda}$. For the $\lambda$-value in the center of the main cardioid of the Mandelbrot set on the right, two of the critical points are fixed. These critical points are symmetrically located under $z \mapsto-z$. At the center of the Mandelbrot set on the left, a pair of symmetric critical points are interchanged by $F_{\lambda}$. Hence these critical points lie on a superstable cycle of period 2 . We shall often encounter this kind of discrepancy when $n$ is odd in the sequel, so we will say that a Mandelbrot set has base period $k$ if the parameter at the center of its main cardioid has a critical point that is mapped to either itself or to its negative (which is also a critical point) by $F_{\lambda}^{k}$, and $k$ is the minimal such iteration. By symmetry, 
it follows that the orbit of this critical point is periodic with period either $k$ or $2 k$. The reason for this somewhat strange choice of terminology is that it will simplify the statement of our main result.

It is known [2] that there are $n-1$ such principal Mandelbrot sets of base period 1 in the degree $2 n$ case and that each of these sets is homeomorphic to the standard quadratic Mandelbrot set. Moreover, for each $\lambda$ in one of these principal Mandelbrot sets, there is an invariant set within the Julia set of $F_{\lambda}$ on which either $F_{\lambda}$ or $F_{\lambda}^{2}$ is topologically conjugate to the corresponding quadratic polynomial on its Julia set. Also apparent in this image are two large Sierpinski holes along the positive and negative imaginary axis. These holes have escape time 3 . In fact, the centers of these Sierpinski holes and the centers of the principal Mandelbrot sets lie along an actual circle in the parameter plane that we call the dividing circle.
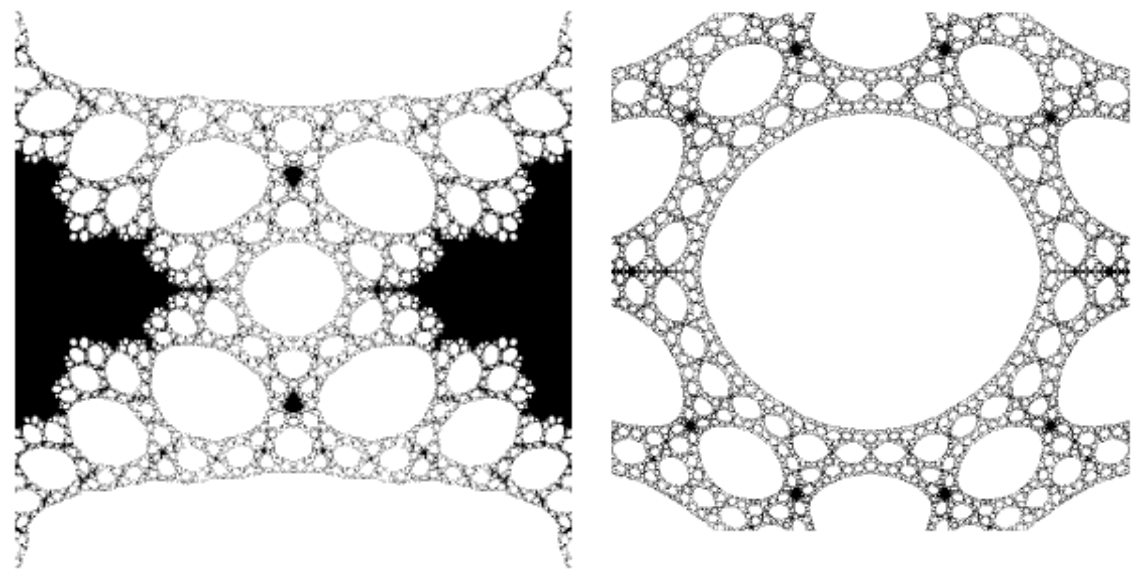

FiguRE 2. Magnifications of the parameter plane for the family $z^{3}+\lambda / z^{3}$ around the McMullen domain.

In Figure 2, we have displayed several magnifications of the region around the McMullen domain in the case $n=3$. In the first image, note that there appear to be four large Sierpinski holes that surround the McMullen domain. These Sierpinski holes are known to have escape time 4. Between the two upper and the two lower Sierpinski holes there appear to be small copies of a Mandelbrot set, while between the two left and two right holes we see the period two bulb of a principal Mandelbrot set and the remainder of the "tail" of this set. In fact, the centers of the main cardioids of the two small Mandelbrot sets are parameters for which two symmetric critical points are interchanged by $F_{\lambda}^{2}$, so we again say that these Mandelbrot sets have base period 2. Thus, there appears to be another curve encircling the McMullen domain that contains four parameter values lying at the centers of Sierpinski holes with escape time 4 and four other parameters for which the critical orbit is periodic of period 2 (under either $F_{\lambda}$ or $F_{\lambda}^{2}$ ).

Inside the closed curve passing through these four Sierpinski holes appears to be another simple closed curve meeting ten Sierpinski holes. Each of these holes has escape time 5. Also, each pair of these holes apparently has either a small copy of a Mandelbrot set or a copy of such a set inside the principal Mandelbrot 
set between them. Each of these Mandelbrot sets has base period 3. Examining the further magnification in Figure 2, there is another closed curve meeting 28 Sierpinski holes with escape time 6 and, inside that curve, an even smaller curve meeting 82 Sierpinski holes with escape time 7. It appears that, in this case, the $k^{\text {th }}$ curve meets exactly $3^{k}+1$ Sierpinski holes with escape time $k+3$ as well as the same number of Mandelbrot sets. We call these curves the rings around the McMullen domain.
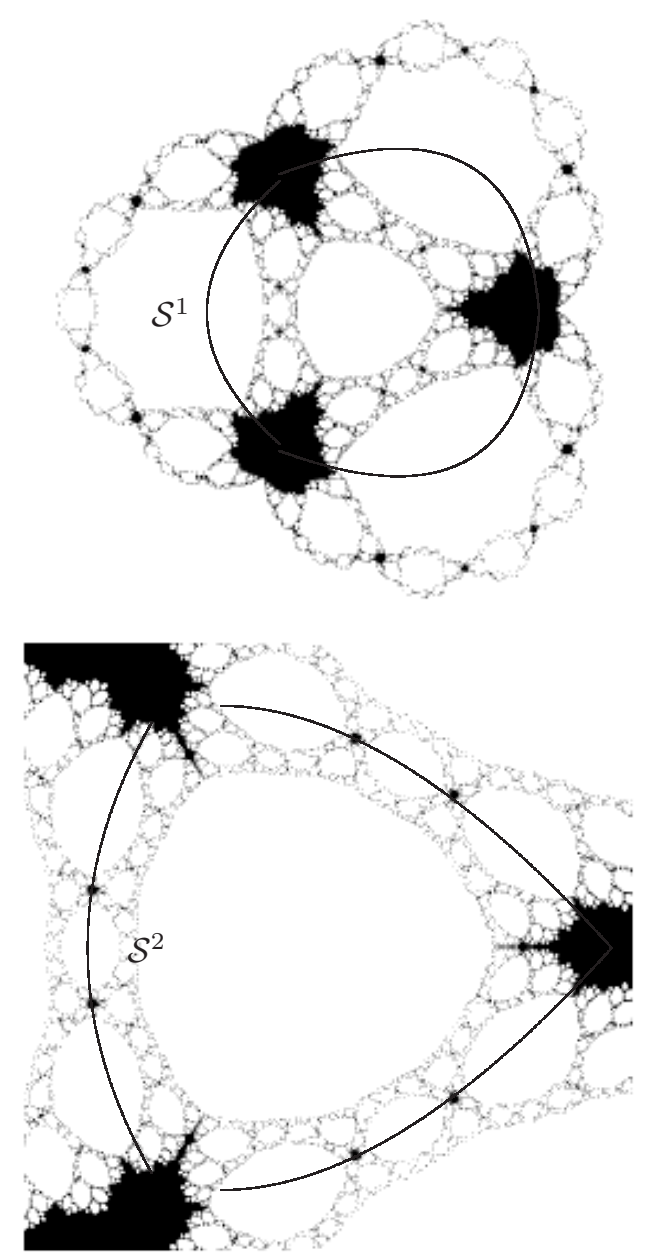

Figure 3 . The curves $\mathcal{S}^{1}$ and $\mathcal{S}^{2}$ in parameter plane for $n=4$.

In Figure 3 we display the parameter plane for $F_{\lambda}$ when $n=4$ as well as a magnification of the region around the McMullen domain. Now there appears to be a closed curve $\mathcal{S}^{1}$ that meets the three principal Mandelbrot sets caught between three large Sierpinski holes, each of which has escape time 3. This is the dividing circle. Inside this curve is another closed curve $\mathcal{S}^{2}$ meeting 9 Sierpinski holes, each with escape time 4 . Between these holes we again see either a smaller copy of a 
Mandelbrot set or a portion of the principal Mandelbrot set. Further inside there is another curve containing 33 holes with escape time 5 .

The following result concerning the existence of these special closed curves in parameter space was proved in [4].

Theorem (Rings Around the McMullen Domain). For each $n \geq 3$, the McMullen domain in the parameter space for the family $z^{n}+\lambda / z^{n}$ is surrounded by infinitely many disjoint simple closed curves (rings) $\mathcal{S}^{d}$ for $d=1,2, \ldots$ having the property that:

(1) Each curve $\mathcal{S}^{d}$ surrounds the McMullen domain as well as $\mathcal{S}^{d+1}$, and the $\mathcal{S}^{d}$ accumulate on the boundary of the McMullen domain as $d \rightarrow \infty$.

(2) The curve $\mathcal{S}^{d}$ contains $\tau_{d}^{n}$ parameter values for which the critical orbits all land on $\infty$ after $d+2$ iterations where

$$
\tau_{d}^{n}=(n-2) n^{d-1}+1 .
$$

(3) The curve $\mathcal{S}^{d}$ also passes through $\tau_{d}^{n}$ superstable parameter values where one of the critical points of $F_{\lambda}$ is periodic of period d (when $n$ is even) or is mapped to either itself or interchanged with its negative by $F_{\lambda}^{d}$ (when $n$ is odd).

Our goal in this paper is to extend this result in two ways. We first prove that, with one exception, each of the superstable parameters lying on these curves is actually the center of a small copy of a Mandelbrot set:

Theorem (Satellite Mandelbrot Sets). For $n \geq 3$ and $d \neq 2$, each of the $\tau_{d}^{n}$ superstable parameter values lying on the curve $\mathcal{S}^{d}$ lie at the center of the main cardioid of a Mandelbrot set with base period $d$. When $d=2$, exactly $n-1$ of these parameters lie at the center of the period 2 bulb of the principal Mandelbrot sets that have base period 1 , while the remaining superstable parameters along $\mathcal{S}^{2}$ lie at the centers of Mandelbrot sets with base period 2.

Our second result applies to the Sierpinski holes. Roesch [15] has shown that, in the case $n=2$, each Sierpinski hole is homeomorphic to a disk and that there is a natural uniformization of this disk taking the center of the hole to the origin of the unit disk in $\mathbb{C}$. This uniformization is similar to the uniformization of the exterior of the Mandelbrot set in [7]. Here we make a minor modification to the methods in Roesch's paper in order to extend this result to the case of any $n \geq 3$ :

Theorem (Structure of Sierpinski Holes). Each Sierpinski hole in parameter space is a simply connected open set in which there is a unique parameter for which the critical orbits all land on $\infty$ at a particular iteration of $F_{\lambda}$.

As a consequence of this result, each of the rings $\mathcal{S}^{d}$ also passes through the centers of $\tau_{d}^{n}$ Sierpinski holes in parameter space with escape time $d+2$, and these Sierpinski holes and baby Mandelbrot sets are arranged in alternate fashion around $\mathcal{S}^{d}$.

More generally, this result allows us to determine the precise number of Sierpinski holes lying in the parameter plane for each value of $n$ :

Corollary. For each $n \geq 3$, there are exactly $(2 n)^{k-3}(n-1)$ Sierpinski holes with escape time $k \geq 3$ in the parameter plane. 


\section{PRior Results}

For the remainder of this paper we shall restrict attention to the family of rational maps given by

$$
F_{\lambda}(z)=z^{n}+\lambda / z^{n}
$$

where $n \geq 3$ since it is known that there is no McMullen domain when $n=1,2$. Our goal in this section is to summarize some of the known results concerning this family that are relevant to our main results.

2.1. Elementary mapping properties. In the dynamical plane, the object of principal interest is the Julia set of $F_{\lambda}$, which we denote by $J\left(F_{\lambda}\right)$. The Julia set is the set of points at which the family of iterates of $F_{\lambda},\left\{F_{\lambda}^{n}\right\}$, fails to be a normal family in the sense of Montel. It is known that $J\left(F_{\lambda}\right)$ is also the closure of the set of repelling periodic points for $F_{\lambda}$ as well as the boundary of the set of points whose orbits escape to $\infty$ under iteration of $F_{\lambda}$ (see [12]).

The point at $\infty$ is a superattracting fixed point for $F_{\lambda}$ and we denote the immediate basin of $\infty$ by $B_{\lambda}$. It is well known that $F_{\lambda}$ is conjugate to $z \mapsto z^{n}$ in a neighborhood of $\infty$ in $B_{\lambda}$. There is also a pole of order $n$ for $F_{\lambda}$ at the origin, so there is a neighborhood of 0 that is mapped into $B_{\lambda}$ by $F_{\lambda}$. If this neighborhood is disjoint from $B_{\lambda}$, then we denote the preimage of $B_{\lambda}$ that contains 0 by $T_{\lambda}$. So $F_{\lambda}$ maps both $B_{\lambda}$ and $T_{\lambda}$ in $n$-to- 1 fashion over $B_{\lambda}$. We call $T_{\lambda}$ the trap door since any orbit that eventually enters the immediate basin of $\infty$ must "fall through" $T_{\lambda}$ enroute to $B_{\lambda}$.

The map $F_{\lambda}$ has $2 n$ other critical points given by $\lambda^{1 / 2 n}$. We call these points the free critical points for $F_{\lambda}$. There are, however, only two critical values for each $F_{\lambda}$, and these are given by $\pm 2 \sqrt{\lambda}$. We denote a free critical point by $c_{\lambda}$ and a critical value by $v_{\lambda}$. The map also has $2 n$ prepoles given by $(-\lambda)^{1 / 2 n}$. Note that all of the critical points and prepoles lie on the circle of radius $|\lambda|^{1 / 2 n}$ centered at the origin. We call this circle the critical circle and denote it by $C_{\lambda}$. A straightforward computation shows that $C_{\lambda}$ is mapped in $2 n$-to- 1 fashion onto the straight line segment connecting the two critical values and passing through the origin. We call this line segment the critical segment. One also checks easily that any other circle centered at the origin is mapped by $F_{\lambda}$ in $n$-to- 1 fashion onto an ellipse surrounding the critical segment whose foci lie at the critical values. In particular, $F_{\lambda}$ maps the open disk outside the critical circle as an $n$ to 1 covering onto the exterior of the critical segment in $\overline{\mathbb{C}} . F_{\lambda}$ maps the interior of the critical circle in similar fashion to the complement of the critical segment.

We call the straight ray connecting the origin to $\infty$ and passing through one of the critical points (resp., prepoles) a critical point ray (resp., prepole ray). Each of the $2 n$ critical point rays is mapped 2-to- 1 onto one of the two straight line segments of the form $t v_{\lambda}$, where $t \geq 1$ and $v_{\lambda}$ is the image of the critical point on this ray. So the image of a critical point ray is a straight ray connecting $v_{\lambda}$ to $\infty$. Each of the prepole rays is mapped 1-to-1 onto the straight line $i t \sqrt{\lambda}$, where $t$ is now any real number. Note that the image of each prepole ray is a straight line that is perpendicular to the line $t v_{\lambda}, t \in \mathbb{R}$, that contains the critical segment as well as the images of all of the critical point rays.

Let $S_{\lambda}$ be an open sector bounded by two prepole rays corresponding to adjacent prepoles on $C_{\lambda}$, so $S_{\lambda}$ is a sector in the plane having angle $2 \pi / 2 n$. We call $S_{\lambda}$ a critical point sector since it contains at its "center" a unique critical point of $F_{\lambda}$. 
Similarly, let $P_{\lambda}$ be the open sector bounded by two critical point rays corresponding to adjacent critical points on $C_{\lambda}$. We call $P_{\lambda}$ a prepole sector. The following result follows immediately from the above:

Proposition (Mapping Properties of $F_{\lambda}$ ).

(1) $F_{\lambda}$ maps each critical point sector 2-to-1 onto the open half plane that is bounded by the image of the prepole lines and contains the critical value that is the image of the unique critical point in the sector.

(2) $F_{\lambda}$ maps each prepole sector 1-to-1 onto the entire plane minus the two half lines $\pm t v_{\lambda}$ where $t \geq 1$.

(3) $F_{\lambda}$ maps the region in both the interior and the exterior of the critical circle onto the complement of the critical segment as an $n$-to-1 covering map.

For more details, we refer to [1].

2.2. Symmetries. We now turn to the symmetry properties of $F_{\lambda}$ in both the dynamical and parameter planes. Let $\beta=\exp (\pi i / n)$ so that $\beta$ is a primitive $2 n^{\text {th }}$ root of unity. Then, for each $j$, we have $F_{\lambda}\left(\beta^{j} z\right)=(-1)^{j} F_{\lambda}(z)$. Hence, if $n$ is even, we have $F_{\lambda}^{2}\left(\beta^{j} z\right)=F_{\lambda}(z)$. Therefore the orbits of $z$ and $\beta^{j} z$ land on the same orbit after two iterations and so have the same eventual behavior for each $j$. If $n$ is odd, the orbits of $F_{\lambda}(z)$ and $F_{\lambda}\left(\beta^{j} z\right)$ are either the same or else they become the negatives of each other after the first iteration. In either case it follows that the orbits of $F_{\lambda}\left(\beta^{j} z\right)$ behave symmetrically under $z \mapsto-z$ for each $j$. Hence the Julia set of $F_{\lambda}$ is symmetric under $z \mapsto \beta z$ and we say that $J\left(F_{\lambda}\right)$ has $2 n$-fold symmetry. In particular, each of the free critical points eventually maps onto the same orbit (in case $n$ is even) or onto one of two symmetric orbits (in case $n$ is odd). Thus these orbits all have the same ultimate behavior. This is why the $\lambda$-plane is a natural parameter plane for each of these families.

Let $H_{\lambda}(z)$ be one of the $n$ involutions given by $H_{\lambda}(z)=\lambda^{1 / n} / z$. Then $F_{\lambda}\left(H_{\lambda}(z)\right)$ $=F_{\lambda}(z)$, so that the Julia set is also preserved by each of these involutions. Note that each $H_{\lambda}$ maps the critical circle to itself and maps circles centered at the origin that lie outside the critical circle to similar circles that lie inside the critical circle. It follows that two such circles, one inside and one outside the critical circle, are mapped $n$-to- 1 onto the same ellipse by $F_{\lambda}$.

The parameter plane (see Figures 2 and 3 ) for $F_{\lambda}$ also possesses several symmetries. First of all, we have

$$
\overline{F_{\lambda}(z)}=F_{\bar{\lambda}}(\bar{z})
$$

so that $F_{\lambda}$ and $F_{\bar{\lambda}}$ are conjugate via the map $z \mapsto \bar{z}$. Therefore the parameter plane is preserved by complex conjugation.

We also have $(n-1)$-fold symmetry in the parameter plane for $F_{\lambda}$. To see this, let $\omega=\exp (2 \pi i /(n-1))$, so $\omega$ is a primitive $(n-1)^{\text {st }}$ root of unity. Then, if $n$ is even, we compute that

$$
F_{\lambda \omega}\left(\omega^{n / 2} z\right)=\omega^{n / 2}\left(F_{\lambda}(z)\right) .
$$

As a consequence, for each $\lambda \in \mathbb{C}$, the maps $F_{\lambda}$ and $F_{\lambda \omega}$ are conjugate under the linear map $z \mapsto \omega^{n / 2} z$. In particular, when $\lambda \in \mathbb{R}^{+}$, the positive real axis is preserved by $F_{\lambda}$, and so it follows that the straight ray $\omega^{n / 2} \cdot \mathbb{R}^{+}$is preserved by $F_{\lambda \omega}$.

When $n$ is odd, the situation is a little different. We now have

$$
F_{\lambda \omega}\left(\omega^{n / 2} z\right)=-\omega^{n / 2}\left(F_{\lambda}(z)\right)
$$


Since $F_{\lambda}(-z)=-F_{\lambda}(z)$, we therefore have that $F_{\lambda \omega}^{2}$ is conjugate to $F_{\lambda}^{2}$ via the map $z \mapsto \omega^{n / 2} z$. This means that the dynamics of $F_{\lambda}$ and $F_{\lambda \omega}$ are "essentially" the same, though subtly different. For example, if $F_{\lambda}$ has a fixed point, then under the conjugacy, this fixed point and its negative are mapped to a 2-cycle for $F_{\lambda \omega}$. The line $\omega^{n / 2} \cdot \mathbb{R}^{+}$is no longer invariant under $F_{\lambda \omega}$ when $\lambda \in \mathbb{R}^{+}$but rather it is interchanged with $-\omega^{n / 2} \cdot \mathbb{R}^{+}$. In any event, it follows that the behavior of $F_{\lambda}$ for $\lambda \in \omega^{j} \cdot \mathbb{R}^{+}$is essentially the same as that of maps with corresponding positive real parameters. We therefore call the rays $\omega^{j} \cdot \mathbb{R}^{+}$the symmetry axes in parameter space.

To summarize the symmetry properties of $F_{\lambda}$, we have:

Proposition (Symmetries in the dynamical and parameter plane). The dynamical plane for $F_{\lambda}$ is symmetric under the map $z \mapsto \beta z$ where $\beta$ is a primitive $2 n^{\text {th }}$ root of unity. The parameter plane is symmetric under both $z \mapsto \bar{z}$ and $z \mapsto \omega z$ where $\omega$ is a primitive $(n-1)^{\text {st }}$ root of unity.

2.3. Rings in parameter plane. For each $n$, let $\lambda^{*}$ be the unique real solution to the equation

$$
\left|v_{\lambda}\right|=2|\sqrt{\lambda}|=|\lambda|^{1 / 2 n}=\left|c_{\lambda}\right| \text {. }
$$

A computation shows that

$$
\lambda^{*}=\left(\frac{1}{4}\right)^{\frac{n}{n-1}} .
$$

The circle of radius $\lambda^{*}$ plays an important role in the parameter plane, for if $\lambda$ lies on this circle, it follows that both of the critical values lie on the critical circle for $F_{\lambda}$. We call the circle of radius $\lambda^{*}$ in parameter plane the dividing circle. We will be primarily concerned in later sections with values of the parameter that lie on or inside the dividing circle. In a subsequent paper we shall describe the very different structure of the parameter plane in the exterior of the dividing circle.

The dividing circle plays the role of the ring $\mathcal{S}^{1}$ in parameter plane. If $\lambda$ lies on this circle, then both $v_{\lambda}$ and $c_{\lambda}$ lie on the same circle (the critical circle) in the dynamical plane. As $\lambda$ winds once around the dividing circle in the counterclockwise direction beginning on the real axis, the critical points and prepoles of $F_{\lambda}$ (given by $\lambda^{1 / 2 n}$ and $(-\lambda)^{1 / 2 n}$ respectively) each wind $1 / 2 n$ of a turn around the critical circle, while the critical values $( \pm 2 \sqrt{\lambda})$ each wind one half of a turn around the critical circle, all monotonically in the counterclockwise direction. Hence there are $n-1$ special parameter values on the dividing circle for which a critical point of the corresponding map equals a critical value, so for these special $\lambda$-values we have a superattracting fixed point or 2-cycle for $F_{\lambda}$. There are also $n-1$ other parameters on this circle for which the critical value is a prepole, so these are centers of a Sierpinski hole with escape time 3.

For the rest of this paper, we shall restrict attention to parameter values that lie inside the dividing circle in parameter space. For these parameters, we have that both critical values lie strictly inside the critical circle, so the entire critical segment lies inside this circle as well. Since $F_{\lambda}$ maps the region outside the critical circle as an $n$-to- 1 covering onto the complement of the critical segment, it follows that there is a simple closed curve that is mapped by $F_{\lambda} n$-to- 1 to the critical circle. Denote this curve by $\gamma_{1}$. Then $F_{\lambda}$ maps the exterior of $\gamma_{1}$ as an $n$-to- 1 covering onto the exterior of the critical circle, so there is another simple closed curve $\gamma_{2}$ lying outside $\gamma_{1}$ that is mapped $n$-to- 1 to $\gamma_{1}$. Continuing in this manner, we find a 
succession of simple closed curves $\gamma_{j}$ having the property that $\gamma_{j}$ lies outside $\gamma_{j-1}$ and $F_{\lambda}$ maps $\gamma_{j}$ as an $n$-to- 1 covering onto $\gamma_{j-1}$ and hence as an $n^{j}$-to- 1 covering onto the critical circle.

We may parametrize the $\gamma_{j}$ as follows. We restrict to the case where $\lambda \notin \mathbb{R}^{+}$so $0<\arg \lambda<2 \pi$. First consider the critical circle. Let $c_{0}=c_{0}(\lambda)$ denote the critical point on the critical circle satisfying $\arg c_{0}=(\arg \lambda) / 2 n$. Then parametrize the critical circle by $\gamma_{0}(\theta)$ where $\gamma_{0}(0)=c_{0}$ and $\gamma_{0}(\theta)$ rotates once around the critical circle in the usual manner in the counterclockwise direction as $\theta$ increases from 0 to $2 \pi$. Note that this parametrization depends analytically on $\lambda$ since $c_{0}$ does. Then parametrize $\gamma_{1}$ by requiring that $F_{\lambda}\left(\gamma_{1}(\theta)\right)=\gamma_{0}(\theta)$. So $\gamma_{1}(\theta)$ is periodic with period $2 n \pi$. Inductively, define $\gamma_{j}(\theta)$ in similar fashion by requiring that $F_{\lambda}\left(\gamma_{j}(\theta)\right)=\gamma_{j-1}(\theta)$ and so $\gamma_{j}(\theta)$ is periodic of period $2^{j} \pi$.

As proved in [4], there is then a unique parameter $\lambda_{\theta}^{j}$ for which the second image of $c_{0}$ is given by $\gamma_{j}(\theta)$. This then gives a parametrization of the portion of the ring $\mathcal{S}^{j}$ in parameter plane lying off $\mathbb{R}^{+}$. In particular, the centers of the baby Mandelbrot sets and the Sierpinski holes in our main results will be such parameters.

2.4. Parameters on the real axis. Since there is a principal Mandelbrot set $\mathcal{M}$ straddling $\mathbb{R}^{+}$, it is well known from real dynamics that there is a sequence of parameters $\lambda_{d} \in \mathbb{R}^{+}$for $d=1,2, \ldots$ having the property that the critical point $c_{0} \in \mathbb{R}^{+}$has period $d$ and the orbit of $c_{0}$ is monotonic along $\mathbb{R}^{+}$in the sense that, for $\lambda=\lambda_{d}$,

$$
0<F_{\lambda}\left(c_{0}\right)<c_{0}=F_{\lambda}^{d}\left(c_{0}\right)<F_{\lambda}^{d-1}\left(c_{0}\right)<F_{\lambda}^{d-2}\left(c_{0}\right)<\cdots<F_{\lambda}^{2}\left(c_{0}\right) .
$$

Because of this monotonicity, these periodic orbits cannot have arisen from a bifurcation from some cycle of lower period (except in the case $d=2$, where this does in fact occur). Consequently each $\lambda_{d}$ (except $\lambda_{2}$ ) lies at the center of the main cardioid of a baby Mandelbrot set lying along the positive real axis. For $\lambda_{1}$, this Mandelbrot set is the principal Mandelbrot set $\mathcal{M}$ alluded to earlier. For each $d>2, \lambda_{d}$ lies at the center of a small copy of a Mandelbrot set contained in the "spine" of $\mathcal{M}$ and we have $\lambda_{d+1}<\lambda_{d}$ for each $d$. The parameter $\lambda_{2}$ lies at the center of the period 2 bulb of $\mathcal{M}$. Also, as $d \rightarrow \infty, \lambda_{d}$ approaches the tip of the tail of $\mathcal{M}$ which is known to lie on the boundary of the McMullen domain. Hence $\mathcal{M}$ extends all the way from the Cantor set locus to the McMullen domain along $\mathbb{R}^{+}$.

As above, let $\omega$ be the primitive $(n-1)^{\text {st }}$ root of unity given by $\exp (2 \pi i /(n-1))$. By the symmetry described above, there is a similar sequence of parameter values $\omega \lambda_{d}$ lying on the line $\omega \cdot \mathbb{R}^{+}$in the parameter plane. For these parameter values, the ray $\omega^{n / 2} \cdot \mathbb{R}^{+}$is invariant if $n$ is even, whereas, if $n$ is odd, $F_{\lambda}$ interchanges $\pm \omega^{n / 2} \cdot \mathbb{R}^{+}$. In this latter case, it follows that $F_{\omega \lambda_{d}}$ has a superattracting cycle of period $2 d$ when $d$ is odd, or a pair of superattracting cycles of period $d$ when $d$ is even.

\section{Baby Mandelbrot sets}

In this section we prove the existence of $(n-2) n^{d-1}+1$ homeomorphic copies of the Mandelbrot set with base period $d \geq 2$ arranged around the ring $\mathcal{S}^{d}$ surrounding the McMullen domain. 
3.1. Polynomial-like maps. The main tool for proving the existence of these sets is the Douady-Hubbard theory of polynomial-like maps. To define these maps, let $U^{\prime}$ and $U$ be open simply connected subsets of $\mathbb{C}$ with $\bar{U}^{\prime} \subset U$. An analytic map $G: U^{\prime} \rightarrow U$ is called a polynomial-like map (of degree two) if $G$ is proper of degree two (so each point in $U$ has exactly two preimages in $U^{\prime}$ when counted with multiplicity). It follows that such a polynomial-like map has a unique critical point in $U^{\prime}$.

Now suppose we have a family of polynomial-like maps given by $G_{\mu}: U_{\mu}^{\prime} \rightarrow U$. Usually the range of such a family also depends on the parameter, but this will not be the case for us. We assume that:

(1) $G_{\mu}$ depends analytically on $\mu$ and the parameters $\mu$ lie in a closed disk $W$.

(2) The set $U_{\mu}^{\prime}$ depends continuously on $\mu$.

(3) For $\mu$ in $\partial W, v_{\mu}$ lies in $U-U_{\mu}^{\prime}$, where $c_{\mu}$ is the critical point of $G_{\mu}$ in $U_{\mu}^{\prime}$ and $v_{\mu}=G_{\mu}\left(c_{\mu}\right)$.

(4) As $\mu$ rotates once around the boundary of $W$, the winding number of $v_{\mu}-c_{\mu}$ is \pm 1 .

Under these conditions, Douady and Hubbard [8] have shown that there is a homeomorphic copy of the Mandelbrot set lying in $W$. In addition, if $\mu$ lies in this Mandelbrot set, then the set of points in $U_{\mu}^{\prime}$ whose orbits under $G_{\mu}$ never leave $U_{\mu}^{\prime}$ is homeomorphic to the filled Julia set of the quadratic polynomial corresponding to $G_{\mu}$ under the above homeomorphism.

In the case of the families $F_{\lambda}$ when $n$ is odd, we sometimes need a slight modification of this result. Recall that, in this case, we have $F_{\lambda}(-z)=-F_{\lambda}(z)$. So suppose that the functions $G_{\mu}$ above also have this property. Let $E_{\mu}(z)=-G_{\mu}(z)$. Then $E_{\mu}^{2}=G_{\mu}^{2}$ so that orbits of $E_{\mu}$ and $G_{\mu}$ agree after every second iteration. In addition to the four assumptions above, suppose also that, for each $\mu$, the open sets $U_{\mu}$ have the property that:

(1) $G_{\mu}\left(U_{\mu}\right)$ is disjoint from $U_{\mu}$;

(2) but the family of maps $E_{\mu}$ is a polynomial-like family for $\mu \in W$.

Then, as above, there is a Mandelbrot set in $W$ for the family $E_{\mu}$. Hence there is also a Mandelbrot set for the family $G_{\mu}$ in $W$. The only difference here is that the actual periods of the bulbs in the Mandelbrot set for $G_{\mu}$ are twice the periods of the corresponding bulbs for $E_{\mu}$.

3.2. Existence of baby Mandelbrot sets. In this section we prove that for each $d \geq 2$ there are $(n-2) n^{d-1}+1$ baby Mandelbrot sets with base period $d$ whose centers lie at the superstable parameters on the $\operatorname{ring} \mathcal{S}^{d}$ (with the special exception in the case $d=2$ noted earlier).

Recall that $\omega=\exp (2 \pi i /(n-1))$. As we showed earlier, the parameter plane is symmetric under the rotation $\lambda \mapsto \omega \lambda$. From the results of section 2.4, we know that, for each $d \geq 3$, there is a unique parameter value $\lambda_{d} \in \mathbb{R}^{+}$that lies at the center of a baby Mandelbrot set that has base period $d$ and whose center lies in $\mathcal{S}^{d} \cap \mathbb{R}^{+}$. For $d=1$, the parameter value $\lambda_{1}$ lies at the center of the principal Mandelbrot set along $\mathbb{R}^{+}$; for $d=2$, the parameter value $\lambda_{2}$ lies at the center of the period 2 bulb of this Mandelbrot set. The parameters $\omega^{j} \lambda_{d}$ have similar properties on the other symmetry axes, except that the periods may be $2 d$ in certain cases when $n$ is odd. This gives $n-1$ baby Mandelbrot sets of base period $d$ whose centers lie at the intersection of $\mathcal{S}^{d}$ with one of the symmetry axes, so we need only 
prove the existence of

$$
(n-2) \cdot n^{d-1}-n+2=(n-2)\left(n^{d-1}-1\right)
$$

additional such sets. By the symmetry in the parameter plane, it suffices to restrict attention to the sector in parameter plane given by $0<\arg \lambda<2 \pi /(n-1)$. Since this sector represents $1 /(n-1)$ of the parameter plane, we shall therefore show that there exists

$$
\kappa(d)=(n-2)\left(n^{d-1}-1\right) /(n-1)=(n-2)\left(n^{d-2}+n^{d-3}+\ldots+n+1\right)
$$

additional such baby Mandelbrot sets with centers on $\mathcal{S}^{d}$ in the open sector given by $0<\arg \lambda<2 \pi /(n-1)$.

For each $\lambda$ in this sector, there is a unique critical point $c_{0}=c_{0}(\lambda)$ that satisfies $\arg c_{0}=(\arg \lambda) / 2 n$. We denote the remaining critical points by $c_{j}=c_{j}(\lambda)$ where the index $j$ increases as the $c_{j}$ move around the critical circle in the counterclockwise direction. Let $v_{\lambda}=F_{\lambda}\left(c_{2 j}\right)$ so that $-v_{\lambda}=F_{\lambda}\left(c_{2 j+1}\right)$. We similarly define $p_{0}=$ $p_{0}(\lambda)$ to be the prepole satisfying $\arg p_{0}=(\pi+\arg \lambda) / 2 n$ and index the remaining $p_{j}$ as above. So, along the critical circle, we have the following arrangement of critical points and prepoles:

$$
0<\arg c_{0}<\arg p_{0}<\cdots<\arg c_{2 n-1}<\arg p_{2 n-1}<2 \pi .
$$

Of special importance in the sequel are the points given by $F_{\lambda}^{2}\left(c_{j}\right)=F_{\lambda}\left( \pm v_{\lambda}\right)$. We compute that

$$
F_{\lambda}\left( \pm v_{\lambda}\right)=( \pm 2)^{n} \lambda^{n / 2}+\frac{1}{( \pm 2)^{n} \lambda^{\frac{n}{2}-1}},
$$

so this point is independent of which critical value we choose if $n$ is even, while, if $n$ is odd, there are two symmetrically located points. So, to specify this point uniquely, we define the function $G$ by

$$
G(\lambda)=F_{\lambda}^{2}\left(c_{0}\right)=2^{n} \lambda^{n / 2}+\frac{1}{2^{n} \lambda^{\frac{n}{2}-1}} .
$$

Since we have restricted attention to the open sector in the parameter plane given by $0<\arg \lambda<2 \pi /(n-1)$, we always use the square root of $\lambda$ that lies in the region $0<\arg \sqrt{\lambda}<\pi /(n-1)$ to compute $G$. Note that $G$ is defined in the parameter plane but takes values in the dynamical plane.

Let

$$
\nu=\left(\frac{n-2}{n}\right)^{\frac{1}{n-1}}\left(\frac{1}{4}\right)^{\frac{n}{n-1}} \in \mathbb{R}^{+} .
$$

A straightforward computation shows that $G$ has $n-1$ critical points that are given by $\omega^{j} \nu$ for $j=0, \ldots, n-2$. Moreover, $G$ maps the interval $[0, \nu] \subset \mathbb{R}^{+}$ univalently onto $[G(\nu), \infty] \subset \mathbb{R}^{+}$and the segment $\omega \cdot[0, \nu]$ univalently onto the ray $\omega^{n / 2} \cdot[G(\nu), \infty]$. Note that this latter ray lies on the line $\arg z=n \pi /(n-1)$.

Recall that $\lambda^{*}=4^{n /(1-n)}$ is the radius of the dividing circle in parameter plane. Then we have

$$
\nu=\left(\frac{n-2}{n}\right)^{\frac{1}{n-1}}\left(\frac{1}{4}\right)^{\frac{n}{n-1}}<\left(\frac{1}{4}\right)^{\frac{n}{n-1}}=\lambda^{*} .
$$

Consider the restriction of $G$ to the arc along the dividing circle lying in the sector $0<\arg \lambda<2 \pi /(n-1)$. Let $\eta$ denote this arc. If $\lambda \in \eta$, then both $c_{0}(\lambda)$ and $v_{\lambda}=F_{\lambda}\left(c_{0}\right)$ lie on the critical circle for the corresponding $F_{\lambda}$ and we have

$$
0<\arg c_{0}(\lambda)<\arg v_{\lambda}=(\arg \lambda) / 2<\pi /(n-1) .
$$


Since $v_{\lambda}$ lies on the critical circle, it follows that $G(\lambda)=F_{\lambda}\left(v_{\lambda}\right)$ lies on the critical segment for $F_{\lambda}$. By the above, since the critical segment connects $\pm_{v_{\lambda}}$, it is constrained to lie in the region $0<\arg \lambda<\pi /(n-1)$ and the negative of this sector given by $\pi<\arg \lambda<n \pi /(n-1)$. The only exception to this occurs at the special parameter value $\hat{\lambda}=4^{n /(1-n)} \exp (i \pi /(n-1))$. This is the unique parameter on $\eta$ for which $F_{\lambda}^{2}\left(c_{0}\right)=0$, i.e., $\hat{\lambda}$ is the center of a Sierpinski hole with escape time 3 that meets this portion of the dividing circle. Hence $G$ maps $\eta$ strictly outside the sector $n \pi /(n-1) \leq \arg \lambda \leq 2 \pi$ except at $\hat{\lambda}$ which is mapped to the origin.

We next claim that $G$ is univalent on $\eta$. Indeed, since $G(\lambda)$ lies on the critical segment for $F_{\lambda}$ and the argument of this segment, $\arg \lambda / 2$, increases monotonically as $\arg \lambda$ increases from 0 to $2 \pi /(n-1)$, it follows that no two parameters on $\eta$ are mapped to the same point by $G$.

Let $U$ denote the open sector in dynamical plane given by $n \pi /(n-1)<\arg z<$ $2 \pi$. Also let $X$ denote the portion of the sector in parameter plane given by $0<$ $\arg \lambda<2 \pi /(n-1)$ that lies on or inside the dividing circle. The set $X$ is bounded by the segments $\left[0, \lambda^{*}\right]$ and $\omega \cdot\left[0, \lambda^{*}\right]$ together with the arc $\eta$. We have shown that $G$ maps the boundary of $X$ onto a curve in $\overline{\mathbb{C}}$. The map is univalent on the boundary of $X$ with the following exceptions:

(1) On the portion of $\partial X$ in $\mathbb{R}^{+}, G$ maps the interval $\left[\nu, \lambda^{*}\right]$ one-to-one to $\left[G(\nu), G\left(\lambda^{*}\right)\right]$ and, since $\nu$ is a critical point of $G$, there is a second interval of the form $[a, \nu]$ with $a>0$ that is also mapped to the same interval.

(2) On the portion of $\partial X$ in $\omega \cdot \mathbb{R}^{+}$, there are a symmetric pair of intervals mapped to the same interval in $\omega^{n / 2} \cdot \mathbb{R}^{+}$.

By the above, we have that the image of the boundary of $X$ therefore lies either along the boundary of the closed sector $\bar{U}$ or else outside of it. If $\lambda \in X$ is very close to the origin, then

$$
G(\lambda) \approx \frac{1}{2^{n} \lambda^{\frac{n}{2}-1}},
$$

so the argument of $G(\lambda)$ lies between $n \pi /(n-1)$ and $2 \pi$. Since there are no critical points of $G$ in the interior of $X$, it follows that $G$ maps $\bar{X}$ univalently (except on the two intervals above) over a region that completely contains the sector $\bar{U}$. Hence there is a curve $\xi$ in $\bar{X}$ that is mapped onto the union of the two segments $[0, G(\nu)]$ and $\omega^{n / 2} \cdot[0, G(\nu)]$ on the boundary of $\bar{U}$. Note that $\xi$ meets the dividing circle only at the parameter value $\hat{\lambda}$ that is mapped to 0 by $G$, and $\xi$ meets $\mathbb{R}^{+}$at $\nu$ and $\omega \cdot \mathbb{R}^{+}$at $\omega \nu$.

We can now define the subset $W$ of parameter space that will be used in the polynomial-like map construction. Let $W$ be the open region in $X$ bounded by the segments $[0, \nu]$ and $\omega \cdot[0, \nu]$ together with the curve $\xi$ (see Figure 4 ). By the above, $G$ maps the boundary of $W$ univalently onto the union of $\mathbb{R}^{+}, \omega \cdot \mathbb{R}^{+}$, the origin, and $\infty$, i.e., the boundary of $\bar{U}$. We have shown:

Proposition 1. G maps the closure of $W$ univalently onto the closed sector $\bar{U}$ in $\overline{\mathbb{C}}$. Moreover, as $\lambda$ travels once around the boundary of $W, G(\lambda)$ travels once around the boundary of this sector in $\overline{\mathbb{C}}$.

Remark. Notice that the set $W$ does not depend on $\lambda$ or on which of the many Mandelbrot sets that will be produced. This is not a problem as it is the domains of the polynomial-like family that will depend on $\lambda$ as will how they are mapped around the plane under various iterations of $F_{\lambda}$. 


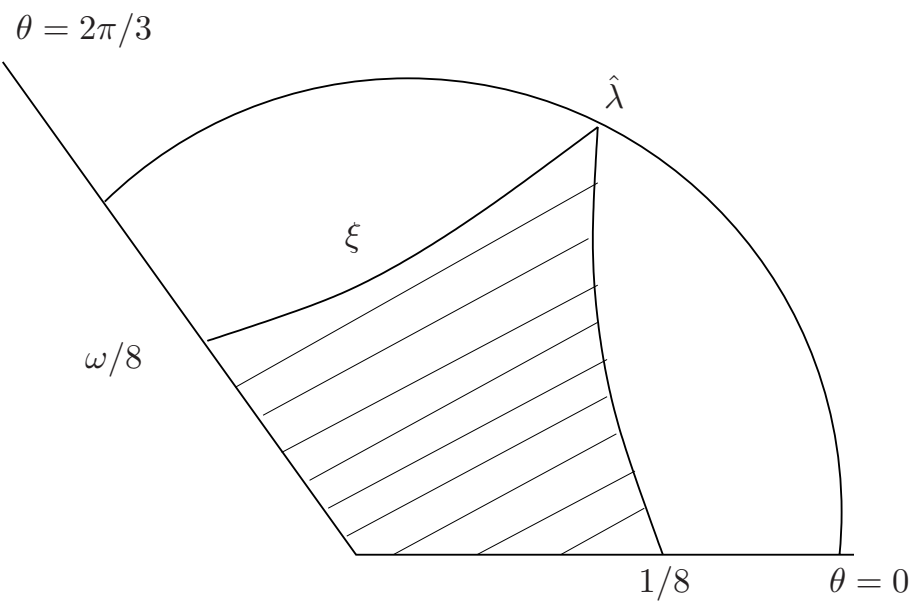

Figure 4 . The region $W$ in the parameter plane when $n=4$. In this case, $\nu=1 / 8$.

We now turn to the construction of the sets $V_{\lambda}^{\prime}$ that will serve as the domains of the functions in the polynomial-like family. We make the further restriction for the rest of this section that $\lambda \in W$. Since $0<\arg \lambda<2 \pi /(n-1)$, we have that the $n-2$ critical points of $F_{\lambda}$ given by $c_{n+2}(\lambda), \ldots, c_{2 n-1}(\lambda)$ always lie in $U$. This follows since

$$
\arg c_{n+2}=\frac{\arg \lambda}{2 n}+\frac{(n+2) \pi}{n}>\frac{(n+2) \pi}{n}>\frac{n \pi}{n-1}
$$

while

$$
\begin{aligned}
\arg c_{2 n-1} & =\frac{\arg \lambda}{2 n}+\frac{(2 n-1) \pi}{n} \\
& <\frac{2 \pi}{2 n}+\frac{(2 n-1) \pi}{n}=2 \pi .
\end{aligned}
$$

A similar computation shows that there are $n-1$ prepoles in $U$ for each $\lambda \in W$, namely $p_{n+1}, \ldots, p_{2 n-1}$. Hence there are $n-2$ critical point sectors that lie in the open sector $U$ for each $\lambda \in W$.

Now fix $d \geq 2$. We shall construct $\kappa(d)$ different sets $V_{\lambda}^{\prime}$ inside $U$ that are each mapped onto $U$ properly of degree two by $F_{\lambda}^{d}$. The difference between these $\kappa(d)$ sets will be determined by how the orbits of the sets $F_{\lambda}^{j}\left(V_{\lambda}^{\prime}\right)$ move through the plane for $0 \leq j<d$.

Let $P_{0}(\lambda)$ denote the closed inner prepole sector containing $p_{0}(\lambda)$, i.e., the sector bounded by the rays through $c_{0}$ and $c_{1}$ and contained on or inside the critical circle. We have $0<\arg z<\pi /(n-1)$ for any $z \in P_{0}(\lambda)$ and $\lambda \in W$ since $0<\arg c_{0}<\arg c_{1}<\pi /(n-1)$ for these $\lambda$-values. Recall that $U$ is the open sector given by

$$
\frac{n \pi}{n-1}<\arg z<2 \pi
$$

Proposition 2. For each $\lambda \in W$, there is a preimage of $U$ under $F_{\lambda}$ that is a simply connected open set in $P_{0}(\lambda)$ and $F_{\lambda}$ maps this preimage univalently onto $U$. The closure of this preimage meets the boundary of $P_{0}(\lambda)$ only at the origin and $p_{0}$. 
Proof. The image of the entire set $P_{0}(\lambda)$ under $F_{\lambda}$ is a half plane bounded by the straight line passing through both critical values and extending to $\infty$ in both directions. This line lies outside of $U$ for each $\lambda$ since $0<\arg v_{\lambda}<\pi /(n-1)$ and $\pi<\arg \left(-v_{\lambda}\right)<n \pi /(n-1)$. The interior of $P_{0}(\lambda)$ is mapped univalently onto one of the two open half planes bounded by this straight line. A computation shows that the image of the prepole line in $P_{0}(\lambda)$ is a straight line that is perpendicular to the straight line passing through the critical values and that lies in the right half plane. Hence it follows that the image half plane contains $U$. Since the boundary of $U$ meets the straight line boundary of this half plane only at the origin and $\infty$, it follows that the closure of the preimage of $U$ in $P_{0}(\lambda)$ meets the boundary of $P_{0}(\lambda)$ only at the origin and $p_{0}$.

Let $V_{\lambda}^{1}$ denote the union of the preimage of $U$ in $P_{0}(\lambda)$ together with the negative of this set. By Proposition $1, G(\lambda)=F_{\lambda}\left(v_{\lambda}\right)$ lies in $U$ for each $\lambda \in W$, so $v_{\lambda}$ lies in the preimage of $U$ in $P_{0}(\lambda)$ under $F_{\lambda}$. Similarly, $-v_{\lambda}$ lies in the other component of $V_{\lambda}^{1}$, so $V_{\lambda}^{1}$ contains both critical values. When $n$ is even, since $F_{\lambda}(-z)=F_{\lambda}(z)$, the negative of the preimage of $U$ is also a preimage of $U$. When $n$ is odd, this set is mapped by $F_{\lambda}$ to $-U$. In this case, the negative of the preimage of $U$ is contained in the inner prepole sector $P_{n}(\lambda)=-P_{0}(\lambda)$ containing the prepole $p_{n}(\lambda)$. As in the previous proposition, this preimage is a simply connected open set and its closure meets the boundary of $P_{n}(\lambda)$ only at the origin and $p_{n}$. Note that, unlike $U$, these two preimage sets depend on $\lambda$. Also, since $0<\arg c_{0}<\arg c_{1}<\pi / n-1$, we have that the sets $P_{0}(\lambda)$ and $P_{n}(\lambda)$ do not meet either of $\pm U$, and so $V_{\lambda}^{1}$ is disjoint from $\pm U$ (see Figure 5 ). We have shown:

Proposition 3. For each $\lambda \in W$, the set $V_{\lambda}^{1}$ is contained in the union of the prepole sectors $P_{0}(\lambda)$ and $P_{n}(\lambda)$ and is disjoint from $\pm U$.

Moreover, we also have:

Proposition 4. Suppose $\lambda \in W$. Then the entire critical segment (excluding the origin) lies in $V_{\lambda}^{1}$.

Proof. Let $t v_{\lambda}$ be a point on the critical segment with $0<|t|<1$. We shall show that $F_{\lambda}\left(t v_{\lambda}\right)$ lies in $U$ for each such $t$ (when $n$ is even) or in $\pm U$ (when $n$ is odd).

First suppose that $n$ is even. Since $v_{\lambda}=2 \sqrt{\lambda}$, we have that

$$
F_{\lambda}\left(t v_{\lambda}\right)=(2 t)^{n} \lambda^{\frac{n}{2}}+\frac{1}{(2 t)^{n} \lambda^{\frac{n}{2}-1}} .
$$

Note that the term

$$
\frac{1}{(2 t)^{n} \lambda^{\frac{n}{2}-1}}
$$

in this sum always lies in $U$. Indeed, we have

$$
0<\arg \lambda^{\frac{n}{2}-1}<\frac{(n-2) \pi}{n-1}
$$

so that

$$
\frac{n \pi}{n-1}<\arg \frac{1}{\lambda^{\frac{n}{2}-1}}<2 \pi
$$




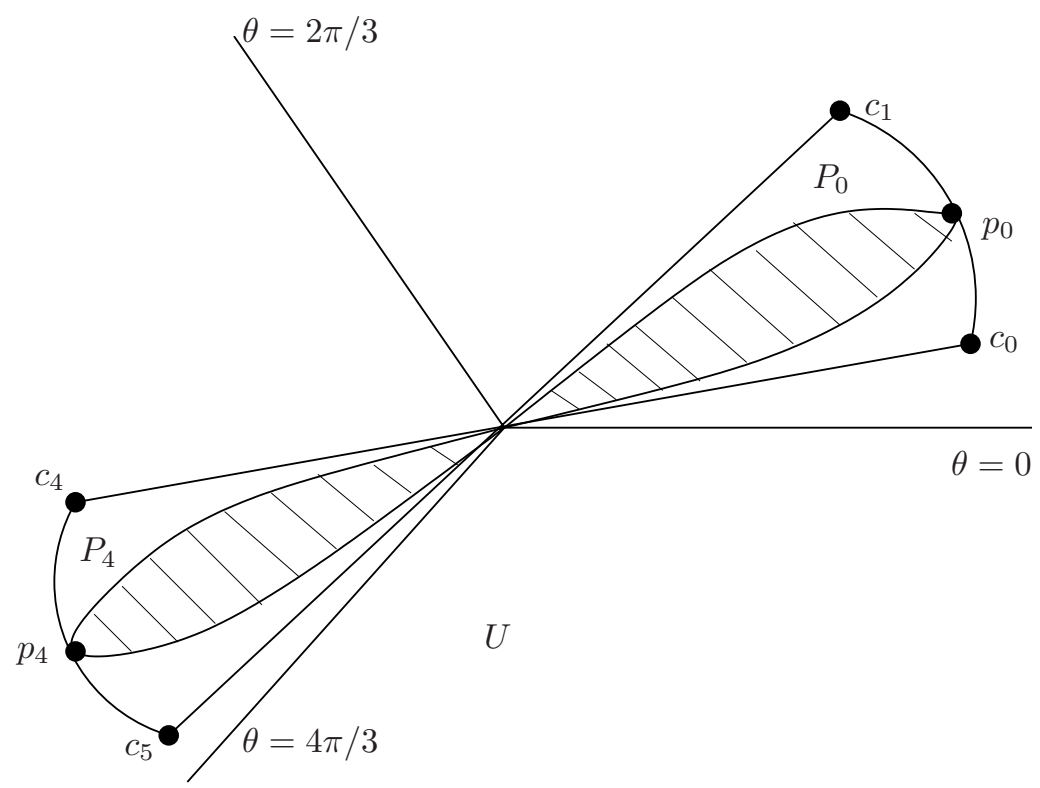

Figure 5. The region $V_{\lambda}^{1}$ in the dynamical plane when $n=4$.

Since $n$ is even, it therefore follows that

$$
\frac{1}{(2 t)^{n} \lambda^{\frac{n}{2}-1}}
$$

lies in $U$ for each $t \neq 0$.

Consider the closed sector bounded by the ray passing through the origin and $1 / 2^{n} \lambda^{\frac{n}{2}-1}$ and also the ray passing through the origin and $F_{\lambda}\left(v_{\lambda}\right)$. Call this sector $Z$. Note that $Z \subset U$ since both $1 / 2^{n} \lambda^{\frac{n}{2}-1}$ and $G(\lambda)=F_{\lambda}\left(v_{\lambda}\right)$ lie in $U$. Also note that, for each $t \neq 0$, the point $1 / 2^{n} t^{n} \lambda^{\frac{n}{2}-1}$ lies on the boundary of $Z$.

We now proceed geometrically. When $t=1$, we think of the term $2^{n} \lambda^{n / 2}$ as a vector in the plane extending from $1 / 2^{n} \lambda^{\frac{n}{2}-1}$ to $F_{\lambda}\left(v_{\lambda}\right)$ since we have $1 / 2^{n} \lambda^{\frac{n}{2}-1}+$ $2^{n} \lambda^{n / 2}=F_{\lambda}\left(v_{\lambda}\right)$. For $|t| \neq 1$, the vector $2^{n} t^{n} \lambda^{n / 2}$ is shorter than $2^{n} \lambda^{n / 2}$ but points in the same direction as $2^{n} \lambda^{n / 2}$. Meanwhile, the point $1 / 2^{n} t^{n} \lambda^{\frac{n}{2}-1}$ lies on the boundary of $Z$ but further away from the origin than $1 / 2^{n} \lambda^{\frac{n}{2}-1}$. Hence the sum of $2^{n} t^{n} \lambda^{n / 2}$ and $1 / 2^{n} t^{n} \lambda^{\frac{n}{2}-1}$ lies in $Z$ and therefore also in $U$. So we have $F_{\lambda}\left(t v_{\lambda}\right) \in U$ for each $t$ with $0<|t| \leq 1$ and $n$ even.

When $n$ is odd, we have that the above proof works for $0<t<1$. For $-1<t<0$, by the $z \mapsto-z$ symmetry, we then have that $F_{\lambda}\left(t v_{\lambda}\right)$ lies in $-U$.

Now consider the entire preimage of $V_{\lambda}^{1}$ under $F_{\lambda}$, not just the preimages lying in $\pm P_{0}(\lambda)$. We denote this preimage by $V_{\lambda}^{2}$. Recall that each critical point sector is mapped two-to-one onto a half plane bounded by the straight line given by $i t v_{\lambda}$ with $t \in \mathbb{R}$, i.e., the line that is the image of the prepole rays and hence perpendicular to the critical segment. Observe that the interiors of the inner prepole sectors $P_{0}(\lambda)$ and $P_{n}(\lambda)$ are each contained in one of these half planes. This follows since

$$
0<\arg c_{0}<\arg c_{1}<\frac{\pi}{n-1} \leq \frac{\pi}{2}<\arg i v_{\lambda}<\frac{(n+1) \pi}{2(n-1)} \leq \pi
$$




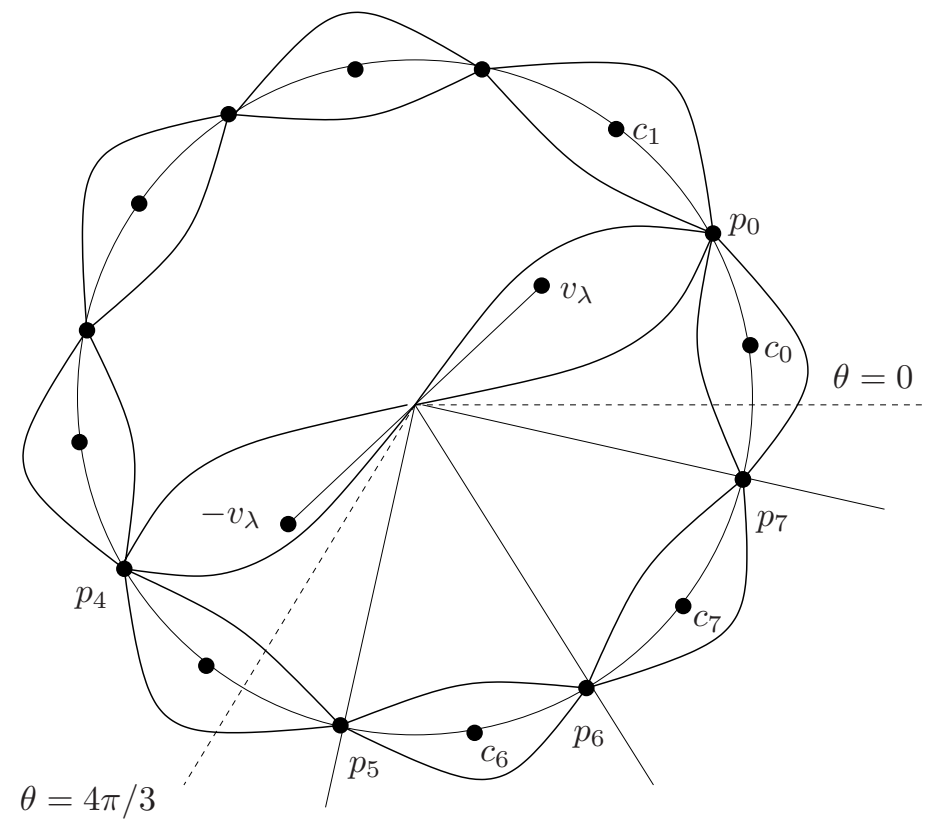

Figure 6 . The region $V_{\lambda}^{2}$ in the dynamical plane together with the critical point sectors containing $c_{7}$ and $c_{8}$ when $n=4$.

for $\lambda \in W$. Therefore each of the two open sets comprising $V_{\lambda}^{1}$ is completely contained in one of these half planes. It then follows that $V_{\lambda}^{2}$ consists of a collection of $2 n$ disjoint open sets, one lying in each critical point sector. Each of these open sets contains the entire portion of the critical circle that lies in this critical point sector since, by Proposition 4, the critical segment minus the origin lies in the image of these sets. Each of these open sets in $V_{\lambda}^{2}$ is also mapped two-to-one onto one of the two components of $V_{\lambda}^{1}$. Note also that the open sets in $V_{\lambda}^{2}$ do not meet $V_{\lambda}^{1}$. This follows since the image of $V_{\lambda}^{1}$ is contained in $\pm U$ whereas the images of the open sets in $V_{\lambda}^{2}$, namely the components of $V_{\lambda}^{1}$, lie in the complement of these sectors by Proposition 3. Since the boundary of $V_{\lambda}^{1}$ meets the boundary of the image of each critical point sector only at the origin, it follows that the boundary of each component of $V_{\lambda}^{2}$ meets the boundary of the critical point sector in which it lies only at the two prepoles lying on the boundary rays of the sector. $V_{\lambda}^{2}$ therefore is a collection of $2 n$ symmetrically arranged open sets whose closures meet only at the prepoles (see Figure 6). To summarize, we have:

Proposition 5. Each of the $2 n$ open sets comprising $V_{\lambda}^{2}$ :

(1) lies in a distinct critical point sector;

(2) is disjoint from $V_{\lambda}^{1}$ and hence disjoint from the critical segment as well;

(3) meets the critical circle in the open arc between adjacent prepoles; and,

(4) is mapped two-to-one onto one of the two components of $V_{\lambda}^{1}$.

Recall that $\gamma_{1}$ is the preimage of the critical circle lying outside $C_{\lambda}$ and that $H_{\lambda}\left(\gamma_{1}\right)$ is the corresponding preimage of the critical circle lying inside $C_{\lambda}$. The set $V_{\lambda}^{2}$ does not meet $\gamma_{1}$ or $H_{\lambda}\left(\gamma_{1}\right)$ since the image of this set does not meet the critical 
circle. (The boundary of $V_{\lambda}^{2}$ does meet $\gamma_{1}$ and $H_{\lambda}\left(\gamma_{1}\right)$, but only at the preimages of the prepoles $p_{0}$ or $p_{n}$.)

3.3. The Case $d=2$. We can now prove the Satellite Mandelbrot Sets theorem in the case $d=2$. We need to prove the existence of $\kappa(2)=n-2$ baby Mandelbrot sets inside $W$ with centers on $\mathcal{S}^{2}$. Consider the $n-2$ critical point sectors that contain the critical points $c_{j}$ where $j=n+2, \ldots, 2 n-1$. We claim that the closures of each of these sectors is contained in $U \cup\{0\}$ for each $\lambda \in W$. Indeed, the union of these sectors is contained in the sector bounded by the prepole rays through $p_{n+1}$ and $p_{2 n-1}$. We have, since $n \geq 3$,

and

$$
\arg p_{n+1}=\frac{\arg \lambda+\pi}{2 n}+\frac{(n+1) \pi}{n}>\frac{(2 n+3) \pi}{2 n} \geq \frac{n \pi}{n-1}
$$

$$
\arg p_{2 n-1}=\frac{\arg \lambda+\pi}{2 n}+\frac{(2 n-1) \pi}{n}<\frac{\left(4 n^{2}-5 n+3\right) \pi}{2 n(n-1)} \leq 2 \pi .
$$

Now fix any $j$ with $n+2 \leq j \leq 2 n-1$. Define $V_{\lambda}^{\prime}$ to be the component of $V_{\lambda}^{2}$ that contains $c_{j}$. We shall show that $F_{\lambda}^{2}$ is a polynomial-like family that takes $V_{\lambda}^{\prime}$ to $U$ for each $\lambda \in W$ when $n$ is even, or to one of $\pm U$ when $n$ is odd.

Suppose first that $n$ is even. By construction, we then have that $F_{\lambda}^{2}$ takes $V_{\lambda}^{\prime}$ onto $U$ in two-to-one fashion. We claim that $\bar{V}_{\lambda}^{\prime}$ is strictly contained inside $U$. This follows since the closure of the critical point sector containing $V_{\lambda}^{\prime}$ is contained in $U \cup\{0\}$ and $\bar{V}_{\lambda}^{\prime}$ is bounded away from 0 in this sector. If $\lambda \in \partial W$, then $F_{\lambda}^{2}\left(c_{j}\right)$ lies on the boundary of $U$ and makes one transit around this boundary as $\lambda$ moves around the boundary of $W$ as shown in Proposition 1 . Hence $F_{\lambda}^{2}\left(c_{j}\right)$ winds once around $V_{\lambda}^{\prime}$ in the region $U-V_{\lambda}^{\prime}$ for these $\lambda$-values. It follows that $F_{\lambda}^{2}$ is a polynomial-like family on $W$. This produces a baby Mandelbrot set with base period 2. By construction, the center of this Mandelbrot set is a parameter for which the critical point $c_{j}$ is periodic with period 2, so this parameter lies on the curve $\mathcal{S}^{2}$. Moreover, since the centers of each of these Mandelbrot sets correspond to different superstable cycles, we have produced $n+1$ such sets, each having centers corresponding to a different critical point among the points $c_{n+2}, \ldots, c_{2 n-1}$.

When $n$ is odd, the image of $V_{\lambda}^{\prime}$ under $F_{\lambda}^{2}$ lies in either $U$ or $-U$. In the former case, the previous arguments apply to produce a baby Mandelbrot set of base period 2 in $W$; in the latter case, by our modification of the Douady-Hubbard theory of polynomial-like maps in section 3.1 , we apply the result to $-F_{\lambda}^{2}$ to produce a baby Mandelbrot set of base period 2. This proves the Satellite Mandelbrot Set theorem in the case $d=2$.

3.4. The Case $d \geq 3$. For the general case $d \geq 3$, we again first restrict attention to the case where $n$ is even. Let $U_{\text {out }}$ denote the portion of the open sector $U$ that lies on or outside the critical circle.

Proposition 6. Suppose $n$ is even. There is a closed subset $Q_{\lambda}$ contained in $U_{\text {out }}$ that has the property that $F_{\lambda}$ maps $Q_{\lambda}$ as a covering onto the set of points satisfying

$$
n \pi+\frac{n \pi}{n-1} \leq \arg z \leq 2 n \pi .
$$

Remark. We interpret this result geometrically as follows: Since

$$
2 n \pi-n \pi-\frac{n \pi}{n-1}=\left(\frac{n-2}{2}\right) \cdot 2 \pi+\left(\frac{n-2}{n-1}\right) \pi,
$$


it follows that $F_{\lambda}$ wraps the region $Q_{\lambda}$ a total of $(n-2) / 2$ full revolutions in the clockwise direction around $\mathbb{C}$ starting at $\mathbb{R}^{+}$, followed by an additional rotation of $(n-2) \pi /(n-1)$ radians. In particular, the image of $Q_{\lambda}$ covers the sector

$$
2 \pi-\left(\frac{n-2}{n-1}\right) \pi=\frac{n \pi}{n-1} \leq \arg z \leq 2 \pi
$$

exactly $n / 2$ times and its complement $(n / 2)-1$ times.

Proof. The boundary of $U_{\text {out }}$ consists of three curves: the portion of the critical circle lying in $\bar{U}$ together with the rays in $\mathbb{R}^{+}$and $\arg z=n \pi /(n-1)$ that extend from the critical circle to $\infty$. We claim that each of these curves is mapped to the complement of $U$ by any $F_{\lambda}$ with $\lambda \in W$, and this image meets $\bar{U}$ only at the origin.

To see this, note first that the critical circle portion of the boundary is wrapped by $F_{\lambda}$ around the critical segment and this segment lies outside of $\bar{U}$ (except at 0 ) for each $\lambda \in W$. The ray $\mathbb{R}^{+}$is mapped to a curve of the form $t^{n}+\lambda / t^{n}$ with $t \geq 0$, so this image resides in the sector $0<\arg z<2 \pi /(n-1)$ which lies in the upper half plane and so is disjoint from $\bar{U} \operatorname{since} \arg \lambda>0$. Finally, if $\arg z=n \pi /(n-1)$, then

$$
\arg z^{n}=\frac{n^{2} \pi}{n-1}=n \pi+\frac{n \pi}{n-1} .
$$

So $z^{n}$ lies along the straight line with argument $n \pi /(n-1)$ since $n$ is even. Also,

$$
-\frac{n \pi}{n-1}<\arg \frac{\lambda}{z^{n}}<\frac{(2-n) \pi}{n-1}
$$

or, adding $2 \pi$ to both sides,

$$
\frac{(n-2) \pi}{n-1}<\arg \frac{\lambda}{z^{n}}<\frac{n \pi}{n-1} .
$$

Hence the sum of these two terms must satisfy

$$
\frac{(n-2) \pi}{n-1}<\arg \left(z^{n}+\frac{\lambda}{z^{n}}\right)<\frac{n \pi}{n-1},
$$

and so the image of this boundary curve lies in a sector to the left of $\bar{U}$. Consequently, the image of each of the boundary curves lies outside $\bar{U}$ (except at 0 ) as claimed.

Now there are no critical points in the interior of $U_{\text {out }}$, so $F_{\lambda}$ is a covering map on this set. Consider the arc of a circle $\nu$ with very large radius that lies in $U_{\text {out }}$. If $z \in \nu$, then

$$
\frac{n \pi}{n-1} \leq \arg z \leq 2 \pi
$$

Then, using the above, since $F_{\lambda}(z) \approx z^{n}$ for $|z|$ large, we have that $F_{\lambda}(\nu)$ is a nearly circular arc that extends from above $\arg z=2 n \pi$ to below

$$
\arg z=\frac{n^{2} \pi}{n-1}=n \pi+\frac{n \pi}{n-1} .
$$

So we define $Q_{\lambda}$ to be the preimage of the region

$$
Y=\left\{z \in \mathbb{C} \mid n \pi+\frac{n \pi}{n-1} \leq \arg z \leq 2 n \pi\right\}
$$

under $F_{\lambda}$. So $Q_{\lambda}$ is a closed subset of $U_{\text {out }}$ and $F_{\lambda}$ takes $Q_{\lambda}$ onto $Y$ as a covering. This completes the proof. 
Recall that $F_{\lambda}$ is an $n$-to- 1 covering map in the exterior of the critical circle that takes this region to the exterior of the critical segment. As we observed in section 2 , since the critical segment lies strictly inside the critical circle, it follows that there is a collection of simple closed curves $\gamma_{j}$ for $j=1,2, \ldots$ lying outside the critical circle and having the property that $F_{\lambda}$ maps $\gamma_{1}$ to the critical circle as an $n$-to- 1 covering and also maps $\gamma_{j+1}$ as an $n$-to- 1 covering onto $\gamma_{j}$ for each $j \geq 1$. So $F_{\lambda}^{j}$ takes $\gamma_{j}$ onto the critical circle as an $n^{j}$-fold covering. These simple closed curves are all disjoint and converge outward to the boundary of the basin of $\infty$ as $j \rightarrow \infty$ and $\gamma_{j}$ is contained in the region inside $\gamma_{j+1}$ for each $j$.

To construct the baby Mandelbrot sets along $\mathcal{S}^{d}$ for $d \geq 3$, we proceed by induction. We continue to assume that $n$ is even. First consider the case $d=3$. Recall that $V_{\lambda}^{2}$ consists of $2 n$ disjoint open sets whose boundaries meet only at the prepoles along the critical circle. By Proposition 5, the closure of $V_{\lambda}^{2}$ contains the entire critical circle and is disjoint from the critical segment.

Since $F_{\lambda}$ is an $n$-to- 1 covering map outside the critical circle, and each component of $V_{\lambda}^{2}$ does not meet the critical segment, each component of $V_{\lambda}^{2}$ therefore has exactly $n$ preimages outside the critical circle. We denote the union of these $2 n^{2}$ preimages by $V_{\lambda}^{3}$. Each component of $V_{\lambda}^{3}$ is disjoint from $V_{\lambda}^{2}$ since, again invoking Proposition 5, the sets $F_{\lambda}\left(V_{\lambda}^{3}\right)=V_{\lambda}^{2}$ and $F_{\lambda}\left(V_{\lambda}^{2}\right)=V_{\lambda}^{1}$ are disjoint. Hence the entire set $V_{\lambda}^{3}$ lies strictly outside the critical circle. Also, each component of $V_{\lambda}^{3}$ meets the curve $\gamma_{1}$ since the image of this set meets the critical circle. Finally, the closure of $V_{\lambda}^{3}$ completely contains $\gamma_{1}$ since $F_{\lambda}\left(\gamma_{1}\right)$ is the critical circle, which lies in the closure of $V_{\lambda}^{2}$.

We claim that there are exactly $(n-2)(n+1)$ components of $V_{\lambda}^{3}$ that lie in the set $Q_{\lambda} \subset U_{\text {out }}$ given by Proposition 6 . To see this, recall that $n-2$ of the $2 n$ components of $V_{\lambda}^{2}$ are strictly contained inside $U$. By Proposition $6, F_{\lambda} \mid Q_{\lambda}$ covers each component of $V_{\lambda}^{2}$ that does not lie in $U$ a total of $(n-2) / 2$ times, while $F_{\lambda} \mid Q_{\lambda}$ covers the $n-2$ components of $V_{\lambda}^{2}$ one additional time. Therefore there are

$$
2 n(n-2) / 2+n-2=(n-2)(n+1)
$$

components of $V_{\lambda}^{3}$ that lie in $Q_{\lambda}$, as claimed. As in the case $d=2$, each of these preimages is strictly contained inside $U_{\text {out }}$ and is mapped by $F_{\lambda}$ onto a component of $V_{\lambda}^{2}$ and then by $F_{\lambda}^{3}$ in two-to-one fashion onto $U$. Note that each of these components varies continuously with $\lambda$ for $\lambda \in W$. Hence we have a family of polynomial-like maps defined on each of these preimages. Since the closures of each of these components is contained inside $U$, we may invoke a similar argument as in the case $d=2$ to show that the critical value of $F_{\lambda}^{3}$ moves once around the boundary of $U$ for parameters in the boundary of $W$. It follows that there is a baby Mandelbrot set in $W$ corresponding to each of these $(n+2)(n-1)$ polynomial-like families.

For $d>3$, we continue in this fashion. There are a total of $2 n^{2}$ components of $V_{\lambda}^{3}$ and $(n-2)(n+1)$ of them lie inside $U$. As above, $F_{\lambda}$ maps $Q_{\lambda}$ over the region $Y$ as a covering, so there are

$$
2 n^{2}(n-2) / 2+(n-2)(n+1)=(n-2)\left(n^{2}+n+1\right)
$$

components of $V_{\lambda}^{4}$ lying in $U$ and $F_{\lambda}^{4}$ is a polynomial-like family on each of them. Continuing in this fashion shows that there are $(n-2)\left(n^{d-1}+\ldots+n+1\right)$ similar components of $V_{\lambda}^{d}$ in $U$ on which $F_{\lambda}^{d}$ is polynomial-like. This concludes the proof when $n$ is even. 
We finally turn to the case where $n$ is odd. Because of the different symmetry present in this case, we need to modify the above proof as well as the consequences of Proposition 6 somewhat. Note first that, since $n$ is odd, $F_{\lambda}$ now maps the ray $\arg z=n \pi /(n-1)$ that bounds $U$ into a different sector, namely the sector

$$
|\arg z|<\frac{\pi}{n-1} .
$$

This follows since $\arg z^{n}=\pi /(n-1)$ whereas

$$
-\frac{\pi}{n-1}<\arg \frac{\lambda}{z^{n}}<\frac{\pi}{n-1}
$$

so the sum of these terms lies in $|\arg z|<\pi /(n-1)$. As a consequence, the image of this boundary curve of $U_{\text {out }}$ lies outside of the region $-U$, not $U$, as before. As in the case where $n$ was even, the image of the portion of the boundary of $U_{\text {out }}$ in $\mathbb{R}^{+}$is mapped to the upper half plane. Note that this curve may meet a portion of $-U$, but this will not affect the definition of $Q_{\lambda}$ below. And, again as before, the image of the critical circle misses both $\pm U$.

We then define $Q_{\lambda}$ as before to be the preimage in $U_{\text {out }}$ of the set

$$
Y=\left\{z \in \mathbb{C} \mid n \pi+\frac{n \pi}{n-1} \leq \arg z \leq 2 n \pi\right\} .
$$

By the above, the straight line boundaries of $U_{\text {out }}$ are mapped outside of the region $Y$. As when $n$ was even, $F_{\lambda}$ wraps $Q_{\lambda}$ a total of $(n-2) / 2$ revolutions around $\mathbb{C}$ in the clockwise direction, starting at $2 n \pi$, and then followed by an additional rotation of $(n-2) \pi /(n-1)$ radians. Since $n$ is odd, this now means that $F_{\lambda}$ wraps $Q_{\lambda}$ around the entire plane in the clockwise direction beginning at $2 n \pi$ a total of $(n-1) / 2$ full revolutions, minus a rotation of $\pi /(n-1)$ radians. Since $F_{\lambda}$ maps the boundaries of $U_{\text {out }}$ beyond the boundaries of $Y$, we again have that $F_{\lambda}$ is a covering map taking the subset $Q_{\lambda}$ onto $Y$.

Now recall that there are $n-2$ components of $V_{\lambda}^{2}$ that are strictly contained in $U$. By symmetry, there are the same number of components in $-U$. Since $V_{\lambda}^{2}$ has $2 n$ components, that leaves four components of $V_{\lambda}^{2}$, two of which intersect each of the sectors between $\pm U$ (though they need not be completely contained in these regions). In particular, only 2 meet the sector $0<\arg z<\pi /(n-1)$ that lies between $U$ and $-U$ in the right half plane. Therefore we have that $F_{\lambda}\left(Q_{\lambda}\right)$ covers $2 n(n-1) / 2-2=(n-2)(n+1)$ of these components. Hence there are the same number of components of $V_{\lambda}^{3}$ lying in $U_{\text {out }}$. So we have $(n-2)(n+1)$ different families of polynomial-like maps $F_{\lambda}^{3}$ defined on these different components.

The proof in the case $d>3$ now proceeds exactly as before, with these modifications. Similarly the proof of the existence of the baby Mandelbrot sets also goes through as above, except that, since $n$ is odd, $F_{\lambda}^{j}$ may take a component of $V_{\lambda}^{j}$ onto $-U$, but the base periods of these Mandelbrot sets are still $j$ since we use $-F_{\lambda}^{j}$ as in the modification of the polynomial-like mapping theorem in section 3.1. This completes the proof of the Satellite Mandelbrot Sets theorem.

\section{SiERPINSKI HOLES}

In this section we investigate the structure of all of the Sierpinski holes in the parameter plane of $F_{\lambda}$, not just those that lie along the rings $\mathcal{S}^{d}$. Let $S$ be such a region. We shall construct a natural analytic homeomorphism $\Phi$ taking $S$ to the complement of the unit disk, $\overline{\mathbb{C}}-\overline{\mathbb{D}}$. $\Phi$ will be a natural generalization of the 
uniformizing map of the exterior of the Mandelbrot set constructed by Douady and Hubbard in [7]. $\Phi$ will also be a straightforward modification of a similar map constructed by Roesch in [15] for the case $n=2$. For this reason we call the map $\Phi$ the Roesch map.

Let $\lambda \in S$. Let $v_{\lambda}=2 \sqrt{\lambda}$ be one of the critical values of $F_{\lambda}$ where we assume $v_{\lambda}$ varies analytically with $\lambda$ over all of $S$. This is possible since $\lambda \neq 0$ in $S$. Let $V_{\lambda}$ be the component of the complement of the Julia set of $F_{\lambda}$ that contains $v_{\lambda}$. Since there are no critical points in the forward orbit of $V_{\lambda}$ until this orbit meets the trap door, there exists $d>0$ such that $F_{\lambda}^{d}$ is an analytic homeomorphism taking $V_{\lambda}$ onto $T_{\lambda}$. Then $H_{\lambda}$ is an analytic homeomorphism taking $T_{\lambda}$ to $B_{\lambda}$. To specify $H_{\lambda}$, we fix a choice of one of the $n$ involutions $H_{\lambda}$ so that $H_{\lambda}$ varies analytically as $\lambda$ ranges over $S$ (see section 2.2). Finally there is another analytic homeomorphism, $\phi_{\lambda}$ (the Böttcher coordinate), taking $B_{\lambda}$ onto $\overline{\mathbb{C}}-\overline{\mathbb{D}}$ and satisfying $\phi_{\lambda}\left(F_{\lambda}(z)\right)=\left(\phi_{\lambda}(z)\right)^{n}$. So the composition $\phi_{\lambda} \circ H_{\lambda} \circ F_{\lambda}^{d}$ is an analytic homeomorphism taking $V_{\lambda}$ to $\overline{\mathbb{C}}-\overline{\mathbb{D}}$.

We now define the Roesch map $\Phi: S \rightarrow \overline{\mathbb{C}}-\overline{\mathbb{D}}$ by

$$
\Phi(\lambda)=\phi_{\lambda}\left(H_{\lambda}\left(F_{\lambda}^{d}\left(v_{\lambda}\right)\right)\right) .
$$

Clearly, $\Phi$ is analytic and maps $S$ into $\overline{\mathbb{C}}-\overline{\mathbb{D}}$. We need to show that $\Phi$ is one-to-one and onto. This will prove that each Sierpinski hole is simply connected. This will also prove that there is a unique $\lambda$ in $S$ that is mapped to $\infty$ with degree one by $\Phi$, so there is a unique $\lambda$-value lying at the center of the Sierpinski hole, i.e., a unique parameter for which the critical points eventually land on $\infty$. Therefore we can give an exact count of the number of Sierpinski holes in the parameter plane (see below).

Recall that $\beta=\exp (\pi i / n)$ so that $\beta^{2 n}=1$ and $F_{\lambda}(\beta z)=-F_{\lambda}(z)$. Hence the Julia set of $F_{\lambda}$ is symmetric under the map $z \mapsto \beta z$.

We now fix $\lambda \in S$. Let $B_{\epsilon}\left(v_{\lambda}\right)$ denote the ball of radius $\epsilon$ about $v_{\lambda}$. We choose $\epsilon$ small enough so that $B_{3 \epsilon}\left(v_{\lambda}\right) \subset V_{\lambda}$. For any $w \in B_{\epsilon}\left(v_{\lambda}\right)$, we define a diffeomorphism $\tau_{w}: \overline{\mathbb{C}} \rightarrow \overline{\mathbb{C}}$ satisfying:

(1) $\tau_{w}$ is the identity map everywhere except on the $2 n$ disjoint disks given by $\beta^{j} \cdot B_{3 \epsilon}\left(v_{\lambda}\right)$ for $j=1, \ldots, 2 n$.

(2) Inside $B_{\epsilon}\left(v_{\lambda}\right), \tau_{w}(z)=z+w-v_{\lambda}$, so $\tau_{w}$ just translates points in this ball by $w-v_{\lambda}$.

(3) In the annulus $B_{3 \epsilon}\left(v_{\lambda}\right)-B_{\epsilon}\left(v_{\lambda}\right), \tau_{w}$ is a $C^{\infty}$ diffeomorphism mapping this annulus to the annulus $B_{3 \epsilon}\left(v_{\lambda}\right)-B_{\epsilon}(w)$ and extending smoothly to the boundaries of $B_{3 \epsilon}\left(v_{\lambda}\right)-B_{\epsilon}\left(v_{\lambda}\right)$.

(4) $\tau_{w}\left(\beta^{j} z\right)=\beta^{j} \tau_{w}(z)$, so this defines $\tau_{w}$ on all the symmetric copies of $B_{3 \epsilon}\left(v_{\lambda}\right)$.

(5) $\tau_{v_{\lambda}}$ is the identity map everywhere and $\tau_{w}$ varies smoothly with $w$.

So, for each $w, \tau_{w}$ is a "bump function" that holomorphically moves $v_{\lambda}$ to $w$ and the symmetric images of $v_{\lambda}$ to the corresponding symmetric images of $w$, and $\tau_{w}$ does nothing outside the balls of radius $3 \epsilon$ around these points.

Let $G_{w}=\tau_{w} \circ F_{\lambda}$. The function $G_{w}$ is not holomorphic, but it is smooth. Note that $G_{w}$ has a superattracting fixed point of order $n$ at $\infty$ and a pole of order $n$ at the origin. In fact, for each $w \in B_{\epsilon}\left(v_{\lambda}\right)$, the basin of $\infty$ for $G_{w}$ is just $B_{\lambda}$ and the trap door is $T_{\lambda}$ since $G_{w}=F_{\lambda}$ on these sets. Indeed, all of the preimages of $B_{\lambda}$ and $T_{\lambda}$ under $F_{\lambda}^{-i}$ and $G_{w}^{-i}$ are the same sets; only the orbits of points within these sets have changed. Also, $G_{w}$ has critical points (that is, points where $G_{w}$ is not locally 
one-to-one) at the points $c_{\lambda}$, but the critical values are now given by $\pm w$ rather than $\pm v_{\lambda}$, and $G_{w}$ has the same $2 n$-fold symmetry as $F_{\lambda}$, i.e., $G_{w}\left(\beta^{j} z\right)=(-1)^{j} G_{w}(z)$.

We may define an ellipse field $\mu_{w}$ on $\overline{\mathbb{C}}$ that is preserved by $G_{w}$ as follows. Let $\mu_{w}$ be the standard complex structure defined everywhere except on all of the preimages of the $2 n$ open sets $\beta^{j} \cdot B_{3 \epsilon}\left(v_{\lambda}\right)$ under $G_{w}^{-k}$ for $k \geq 1$. On these preimages, let $\mu_{w}$ be the pullback by $G_{w}^{-k}$ of the standard complex structure on the sets $\beta^{j} \cdot B_{3 \epsilon}\left(v_{\lambda}\right)$. So, for example, $\mu_{w}$ is the standard complex structure on $B_{3 \epsilon}\left(v_{\lambda}\right)$ but is given on $G_{w}^{-1}\left(B_{3 \epsilon}\left(v_{\lambda}\right)\right)$ by applying $F_{\lambda}^{-1} \circ \tau_{w}^{-1}$ to the standard complex structure on $B_{3 \epsilon}\left(v_{\lambda}\right)$. Note that, by construction, $G_{w}$ preserves the ellipse field $\mu_{w}$ and that $\mu_{w}$ varies smoothly with $w$.

Since for each $j$, the ellipse field $\mu_{w}$ on $F_{\lambda}^{-1}\left(\beta^{j} \cdot B_{3 \epsilon}\left(v_{\lambda}\right)\right)$ is obtained by pulling back the standard complex structure by a composition of a diffeomorphism and a holomorphic map, it follows that $\mu_{w}$ has bounded dilatation on this subset. On subsequent preimages, $\mu_{w}$ is obtained by pulling back this structure by just a holomorphic map, so it follows that $\mu_{w}$ has bounded dilatation on all of $\overline{\mathbb{C}}$. Also, since these pullback maps respect the $z \mapsto \beta z$ symmetry, it follows that $\mu_{w}$ also has this $2 n$-fold symmetry.

Since $\mu_{w}$ has bounded dilatation, we may apply the Measurable Riemann Mapping theorem to straighten $\mu_{w}$. This gives a quasiconformal homeomorphism $h_{w}$ : $\overline{\mathbb{C}} \rightarrow \overline{\mathbb{C}}$ that converts $\mu_{w}$ to the standard complex structure almost everywhere. We may normalize $h_{w}$ so that $h_{w}(\infty)=\infty$ and $h_{w}(0)=0$. Then the map given by $R_{w}=h_{w} \circ G_{w} \circ h_{w}^{-1}$ takes the standard complex structure to itself, so $R_{w}$ is a holomorphic map which is, in fact, a rational map of degree $2 n$. We may further normalize $h_{w}$ by requiring that $h_{w}\left(\beta c_{\lambda}\right)=\beta h_{w}\left(c_{\lambda}\right)$ since we have only specified two conditions on $h_{w}$ so far. This requirement actually implies much more about the symmetry of $h_{w}$.

Lemma. $h_{w}(\beta z)=\beta h_{w}(z)$ for all $z \in \overline{\mathbb{C}}$.

Proof. Suppose $h_{w}(\beta z) \neq \beta h_{w}(z)$ for some $z$. Then the function

$$
g_{w}(z)=\beta^{-1} h_{w}(\beta z)
$$

also straightens the ellipse field $\mu_{w}$ since $z \mapsto \beta z$ preserves $\mu_{w}, h_{w}$ converts $\mu_{w}$ to the standard complex structure, and then $z \mapsto \beta^{-1} z$ preserves the standard structure. But we have $g_{w}(\infty)=\infty, g_{w}(0)=0$, and $g_{w}\left(c_{\lambda}\right)=\beta^{-1} h_{w}\left(\beta c_{\lambda}\right)=h_{w}\left(c_{\lambda}\right)$. So $h_{w}$ and $g_{w}$ agree at $0, \infty$, and $c_{\lambda}$. Therefore $h_{w}(z)=g_{w}(z)$ for all $z \in \overline{\mathbb{C}}$.

The map $R_{w}$ has a pole of order $n$ at the origin and a superattracting fixed point of order $n$ at $\infty$ since $G_{w}$ has these properties. Similarly, since $h_{w}(\beta z)=\beta h_{w}(z)$, it follows that $R_{w}\left(\beta^{j} z\right)=(-1)^{j} R_{w}(z)$. Therefore $R_{w}$ actually assumes the form $F_{\alpha}(z)=z^{n}+\alpha / z^{n}$ for some $\alpha=\alpha(w)$ (see below).

It follows that $h_{w}$ gives a quasiconformal conjugacy between the map $G_{w}$ and $F_{\alpha}$. Note that the basin of $\infty$ for $F_{\alpha}$ is given by $B_{\alpha}=h_{w}\left(B_{\lambda}\right)$ and the trap door for $F_{\alpha}$ is given by $T_{\alpha}=h_{w}\left(T_{\lambda}\right)$ since $B_{\lambda}$ is the basin of $\infty$ and $T_{\lambda}$ is the trap door for $G_{w}$. Moreover, the critical points for $F_{\alpha}$ are given by $h_{w}\left(c_{\lambda}\right)$ while the critical values of $F_{\alpha}$ are given by $\pm h_{w}(w)$. Finally, since the construction of $\mu_{w}$ and $h_{w}$ depends continuously on $w$, it follows that $\alpha(w)$ is a continuous function of $w$ and that $\alpha(w)$ lies in $S$ for each $w$. 
Let $H_{\alpha}$ be the involution that fixes the pair of critical points for $F_{\alpha}$ that correspond under $h_{w}$ to those that $H_{\lambda}$ fixes for $F_{\lambda}$. Then, on $T_{\lambda}$, we have $H_{\alpha} \circ h_{w}=$ $h_{w} \circ H_{\lambda}$.

Now we compute $\Phi(\alpha)$ :

$$
\begin{aligned}
\Phi(\alpha) & =\phi_{\alpha}\left(H_{\alpha}\left(F_{\alpha}^{d}\left(h_{w}(w)\right)\right)\right) \\
& =\phi_{\alpha}\left(H_{\alpha}\left(h_{w}\left(G_{w}^{d}(w)\right)\right)\right) \\
& =\phi_{\alpha}\left(H_{\alpha}\left(h_{w}\left(F_{\lambda}^{d}(w)\right)\right)\right) \\
& =\phi_{\alpha}\left(h_{w}\left(H_{\lambda}\left(F_{\lambda}^{d}(w)\right)\right)\right) .
\end{aligned}
$$

In $B_{\alpha}$, we have

$$
F_{\alpha}=h_{w} \circ F_{\lambda} \circ h_{w}^{-1}
$$

so

$$
\phi_{\lambda}\left(h_{w}^{-1}\left(F_{\alpha}(z)\right)\right)=\phi_{\lambda}\left(F_{\lambda}\left(h_{w}^{-1}(z)\right)\right)=\left(\phi_{\lambda}\left(h_{w}^{-1}(z)\right)\right)^{n} .
$$

Therefore

$$
\phi_{\alpha}=\phi_{\lambda} \circ h_{w}^{-1}
$$

and so

$$
\Phi(\alpha)=\phi_{\lambda}\left(H_{\lambda}\left(F_{\lambda}^{d}(w)\right)\right)
$$

Since $\phi_{\lambda} \circ H_{\lambda} \circ F_{\lambda}^{n}$ is an analytic homeomorphism in a neighborhood of $v_{\lambda}$, it therefore follows that $\Phi$ is one-to-one in a neighborhood of $\lambda$.

Now we show that $\Phi$ is onto. Suppose that $\lambda_{0}$ belongs to $\partial S$. Then we claim that $v_{\lambda_{0}} \notin B_{\lambda_{0}}$. If $v_{\lambda_{0}} \in B_{\lambda_{0}}$, this would imply that $\lambda_{0}$ lies in the Cantor set locus in parameter space, which is an open set. Hence all nearby parameter values would lie in this locus, which cannot happen since $\lambda_{0} \in \partial S$. Consequently, we have a Böttcher coordinate $\phi_{\lambda_{0}}$ defined for $F_{\lambda_{0}}$ on $B_{\lambda_{0}}$ and the functions $\phi_{\lambda}$ vary continuously with $\lambda$ at each $\lambda_{0} \in S \cup \partial S$.

If the map $\Phi$ does not take $S$ onto the set $\overline{\mathbb{C}}-\overline{\mathbb{D}}$, then there is a sequence $\lambda_{j} \in S$ that accumulates on some point in $\partial S$, say $\lambda_{0}$, but $\Phi\left(\lambda_{j}\right)$ accumulates on some point, say $z_{0}$, in the open set $\overline{\mathbb{C}}-\overline{\mathbb{D}}$. By definition of $\Phi$, we have

$$
H_{\lambda_{0}}\left(F_{\lambda_{0}}^{d}\left(v_{\lambda_{0}}\right)\right)=\phi_{\lambda_{0}}^{-1}\left(z_{0}\right) \text {. }
$$

Hence $H_{\lambda_{0}}\left(F_{\lambda_{0}}^{d}\left(v_{\lambda_{0}}\right)\right)$ lies in the basin of $\infty$, so $\lambda_{0} \in S$. This contradiction shows that the sequence $\Phi\left(\lambda_{j}\right)$ cannot accumulate on a point in the interior of $\overline{\mathbb{C}}-\overline{\mathbb{D}}$. This shows that $\Phi$ is onto.

Proposition. Suppose $R$ is a rational map of degree $2 n$ with a single pole of order $n$ at the origin. Suppose also that $R\left(\beta^{j} z\right)=(-1)^{j} R(z)$ where $\beta$ is a primitive $2 n^{\text {th }}$ root of unity. Then $R$ assumes the form

$$
R(z)=z^{n}+\frac{\lambda}{z^{n}}
$$

for some $\lambda \neq 0$.

Proof. We may write $R(z)=P(z) / z^{n}$ where

$$
P(z)=a_{2 n} z^{2 n}+\ldots+a_{1} z+a_{0} .
$$


We may first conjugate $R$ by the linear map $z \mapsto a_{2 n}^{1 /(n-1)} z$ so that we may assume that the coefficient $a_{2 n}=1$. Then the equation $R(\beta z)=-R(z)$ implies that

$$
a_{2 n-1} \beta^{2 n-1} z^{2 n-1}+\ldots+a_{2} \beta^{2} z^{2}+a_{1} \beta z=a_{2 n-1} z^{2 n-1}+\ldots+a_{2} z^{2}+a_{1} z
$$

for all $z \in \mathbb{C}$. Therefore $a_{j}=0$ for $j=1, \ldots, 2 n-1$.

Recall that a Sierpinski hole $S$ has escape time $k$ if the orbit of each critical point of $F_{\lambda}$ lands in $B_{\lambda}$ at the $k^{\text {th }}$ iteration for some and hence all $\lambda \in S$.

Proposition. There are exactly $2^{k-3} n^{k-3}(n-1)$ Sierpinski holes with escape time $k$ in the parameter plane for $F_{\lambda}$ for each $k \geq 3$.

Proof. Define the functions $P_{2}(\lambda)$ and $Q_{2}(\lambda)$ by

$$
\frac{P_{2}(\lambda)}{Q_{2}(\lambda)}=F_{\lambda}^{2}\left(c_{\lambda}\right)=2^{n} \lambda^{\frac{n}{2}}+\frac{1}{2^{n} \lambda^{\frac{n}{2}-1}}=\frac{2^{2 n} \lambda^{n-1}+1}{2^{n} \lambda^{\frac{n}{2}-1}} .
$$

So $P_{2}$ is a polynomial in $\lambda$ of degree $n-1$ with $n-1$ distinct nonzero roots. $Q_{2}$ is not a polynomial, but the only root of $Q_{2}$ is 0 . Note that $P_{2}$ and $Q_{2}$ have no common factors. Consequently, there are $n-1$ distinct $\lambda$-values for which $F_{\lambda}^{2}\left(c_{\lambda}\right)=0$. Since there is a unique $\lambda$-value that lands on 0 in each Sierpinski hole, it then follows that there are exactly $n-1$ Sierpinski holes with escape time 3 .

Now define

$$
\frac{P_{3}(\lambda)}{Q_{3}(\lambda)}=F_{\lambda}^{3}\left(c_{\lambda}\right)=\frac{P_{2}^{2 n}+\lambda Q_{2}^{2 n}}{\left(P_{2} Q_{2}\right)^{n}}
$$

We have that $P_{2}^{2 n}$ is a polynomial in $\lambda$ of degree $2 n(n-1)$. Also $\lambda Q_{2}^{2 n}$ is a polynomial of degree $n^{2}-2 n+1$, so the degree of $\lambda Q_{2}^{2 n}$ is less than the degree of $P_{2}^{2 n}$. Hence the numerator $P_{3}$ is a polynomial of degree $2 n(n-1)$. In the denominator, $Q_{3}$ is the product of the polynomial $P_{2}^{n}$ of degree $n(n-1)$ and the term

$$
\lambda^{((n / 2)-1) n},
$$

so the degree of $Q_{3}$ is

$$
n(n-1)+n\left(\frac{n}{2}\right)
$$

Therefore $Q_{3}$ has smaller degree than $P_{3}$.

Note that $P_{3}$ and $Q_{3}$ have no common roots. This follows since the roots of $P_{2}$ and $Q_{2}$ are distinct, as we saw earlier. Hence any root of the denominator $P_{2}^{n} Q_{2}^{n}$ is a root of only one of $P_{2}$ or $Q_{2}$. Therefore only one of the two terms in the numerator vanishes at this root, so $P_{3}$ is nonzero at this point. Therefore we have that there are $2 n(n-1) \lambda$-values counted with multiplicity for which $F_{\lambda}^{3}\left(c_{\lambda}\right)=0$. However, since there is a unique $\lambda$-value that lands on 0 in each Sierpinski hole, it follows that none of these roots are multiple roots. Therefore there are exactly $2 n(n-1)$ Sierpinski holes with escape time 4.

For the general case, we proceed by induction. For $k \geq 3$ let

$$
\frac{P_{k}(\lambda)}{Q_{k}(\lambda)}=F_{\lambda}^{k}\left(c_{\lambda}\right) .
$$


Assume that

(1) $P_{k}$ is a polynomial in $\lambda$ of degree $(2 n)^{k-2}(n-1)$;

(2) $Q_{k}$ is the product of a polynomial in $\lambda$ of degree $\left(2^{k-2}-1\right) n^{k-2}(n-1)$ and the expression

$$
\lambda^{((n / 2)-1) n^{k-2}}
$$

(3) $P_{k}$ and $Q_{k}$ have no common roots.

Then $P_{k+1}=P_{k}^{2 n}+\lambda Q_{k}^{2 n}$. Now $P_{k}^{2 n}$ is a polynomial in $\lambda$ of degree

$$
(2 n)^{k-1}(n-1)=2^{k-1} n^{k}-2^{k-1} n^{k-1} .
$$

Also, $Q_{k}^{2 n}$ is the product of a polynomial in $\lambda$ of degree

$$
2\left(2^{k-2}-1\right) n^{k-1}(n-1)
$$

and

$$
\lambda^{(n-2) n^{k-1}}
$$

which is also a polynomial. So $Q_{k}^{2 n}$ is a polynomial of degree

$$
2\left(2^{k-2}-1\right) n^{k-1}(n-1)+(n-2) n^{k-1}=\left(2^{k-1}-1\right) n^{k}-2^{k-1} n^{k-1} .
$$

Hence $\lambda Q_{k}^{2 n}$ has degree

$$
\left(2^{k-1}-1\right) n^{k}-2^{k-1} n^{k-1}+1
$$

which is smaller than the degree of $P_{k}^{2 n}$. Hence the numerator $P_{k+1}$ is a polynomial of degree $2^{k-1} n^{k-1}(n-1)$.

In the denominator we have $Q_{k+1}=P_{k}^{n} Q_{k}^{n}$ so $Q_{k+1}$ is the product of polynomials of degree $(2 n)^{k-2}(n)(n-1)$ and degree $\left(2^{k-2}-1\right) n^{k-1}(n-1)$ as well as the expression

$$
\lambda^{((n / 2)-1) n^{k-1}} \text {. }
$$

The product of the two polynomials has degree

$$
(2 n)^{k-2}(n)(n-1)+\left(2^{k-2}-1\right) n^{k-1}(n-1)=\left(2^{k-1}-1\right) n^{k-1}(n-1)
$$

so $Q_{k+1}$ is the product of a polynomial of degree $\left(2^{k-1}-1\right) n^{k-1}(n-1)$ and the term

$$
\lambda^{((n / 2)-1) n^{k-1}} .
$$

So the degree of $Q_{k+1}$ is

$$
\left(2^{k-1}-1\right) n^{k-1}(n-1)+n^{k-1}\left(\frac{n}{2}-1\right)=2^{k-1} n^{k-1}(n-1)-\frac{n^{k}}{2} .
$$

Therefore the degree of $P_{k+1}$ is larger than $Q_{k+1}$. By induction, $P_{k}$ and $Q_{k}$ have no common roots. Hence any root of the denominator is a root of only one of the two terms in the numerator, and so the numerator cannot vanish at such a point. Hence there are $2^{k-1} n^{k-1}(n-1)$ distinct roots of the equation $F_{\lambda}^{k+1}\left(c_{\lambda}\right)=0$. As above, each of these roots is a center of a Sierpinski hole and we have shown that there is a unique such center in each hole. This proves that there are exactly $2^{k-1} n^{k-1}(n-1)$ Sierpinski holes with escape time $k+2$, or $2^{k-3} n^{k-3}(n-1)$ Sierpinski holes with escape time $k$ 


\section{REFERENCES}

1. Blanchard, P., Devaney, R. L., Look, D. M., Seal, P., and Shapiro, Y., Sierpinski Curve Julia Sets and Singular Perturbations of Complex Polynomials. Ergodic Theory and Dynamical Systems 25 (2005), 1047-1055. MR2158396 (2006d:37087)

2. Devaney, R. L., Baby Mandelbrot Sets Adorned with Halos in Families of Rational Maps. In Complex Dynamics: Twenty Five Years After the Appearance of the Mandelbrot Set, Contemporary Math 396 (2006), 37-50. MR2209085 (2006k:37131)

3. Devaney, R. L., Structure of the McMullen Domain in the Parameter Planes for Rational Maps. Fundamenta Mathematicae 185 (2005), 267-285. MR2161407 (2006c:37046)

4. Devaney, R. L. and Marotta, S., The McMullen Domain: Rings Around the Boundary. Trans. Amer. Math. Soc. 359 (2007), no. 7, 3251-3273 (electronic). MR2299454

5. Devaney, R. L. and Look, D. M., A Criterion for Sierpinski Curve Julia Sets. Spring Topology and Dynamical Systems Conference. Topology Proc. 30 (2006), no. 1, 163-179. MR2280665

6. Devaney, R. L., Look, D. M., and Uminsky, D., The Escape Trichotomy for Singularly Perturbed Rational Maps. Indiana University Mathematics Journal 54 (2005), 1621-1634. MR2189680 (2006i:37105)

7. Douady, A. and Hubbard, J., Etude Dynamique des Polynômes Complexes. Publ. Math. D’Orsay (1984).

8. Douady, A., and Hubbard, J. On the Dynamics of Polynomial-like Mappings. Ann. Sci. ENS Paris 18 (1985), 287-343. MR816367 (87f:58083)

9. Mane, R., Sad, P., and Sullivan, D., On the Dynamics of Rational Maps. Ann. Sci. ENS Paris 16 (1983), 193-217. MR732343 (85j:58089)

10. McMullen, C., Automorphisms of Rational Maps. Holomorphic Functions and Moduli. Vol. 1. Math. Sci. Res. Inst. Publ. 10. Springer, New York, 1988. MR955807 (89m:58187)

11. McMullen, C., The Classification of Conformal Dynamical Systems. Current Developments in Mathematics. Internat. Press, Cambridge, MA, (1995) 323-360. MR1474980 (98h:58162)

12. Milnor, J., Dynamics in One Complex Variable. Third Edition. Princeton University Press. MR2193309 (2006g:37070)

13. Milnor, J. and Tan Lei, A "Sierpinski Carpet" as Julia Set. Appendix F in Geometry and Dynamics of Quadratic Rational Maps. Experiment. Math. 2 (1993), 37-83. MR1246482 (96b:58094)

14. Petersen, C. and Ryd, G., Convergence of Rational Rays in Parameter Spaces, The Mandelbrot set: Theme and Variations, London Mathematical Society, Lecture Note Series 274, Cambridge University Press, 161-172, 2000. MR1765088 (2001f:37057)

15. Roesch, P., On Capture Zones for the Family $f_{\lambda}(z)=z^{2}+\lambda / z^{2}$. In Dynamics on the Riemann Sphere, European Mathematical Society, (2006), 121-130.

16. Whyburn, G. T., Topological Characterization of the Sierpinski Curve. Fundamenta Mathematicae 45 (1958), 320-324. MR0099638 (20:6077)

Department of Mathematics, Boston University, 111 Cummington Street, Boston, MassachusetTs 02215 